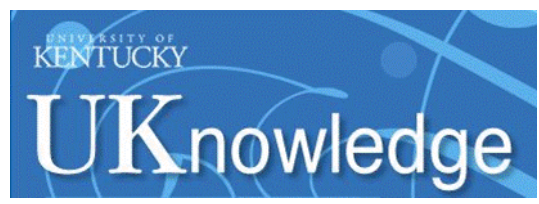

University of Kentucky

UKnowledge

2019

\title{
QUANTIFICATION OF PAPILLARY MUSCLE MOTION AND MITRAL REGURGITATION AFTER MYOCARDIAL INFARCTION
}

Connor R. Ferguson

University of Kentucky, cferguson@uky.edu

Author ORCID Identifier:

(iD) https://orcid.org/0000-0003-2489-2237

Digital Object Identifier: https://doi.org/10.13023/etd.2019.215

Right click to open a feedback form in a new tab to let us know how this document benefits you.

\section{Recommended Citation}

Ferguson, Connor R., "QUANTIFICATION OF PAPILLARY MUSCLE MOTION AND MITRAL REGURGITATION AFTER MYOCARDIAL INFARCTION" (2019). Theses and Dissertations--Mechanical Engineering. 136. https://uknowledge.uky.edu/me_etds/136

This Master's Thesis is brought to you for free and open access by the Mechanical Engineering at UKnowledge. It has been accepted for inclusion in Theses and Dissertations--Mechanical Engineering by an authorized administrator of UKnowledge. For more information, please contact UKnowledge@lsv.uky.edu. 


\section{STUDENT AGREEMENT:}

I represent that my thesis or dissertation and abstract are my original work. Proper attribution has been given to all outside sources. I understand that I am solely responsible for obtaining any needed copyright permissions. I have obtained needed written permission statement(s) from the owner(s) of each third-party copyrighted matter to be included in my work, allowing electronic distribution (if such use is not permitted by the fair use doctrine) which will be submitted to UKnowledge as Additional File.

I hereby grant to The University of Kentucky and its agents the irrevocable, non-exclusive, and royalty-free license to archive and make accessible my work in whole or in part in all forms of media, now or hereafter known. I agree that the document mentioned above may be made available immediately for worldwide access unless an embargo applies.

I retain all other ownership rights to the copyright of my work. I also retain the right to use in future works (such as articles or books) all or part of my work. I understand that I am free to register the copyright to my work.

\section{REVIEW, APPROVAL AND ACCEPTANCE}

The document mentioned above has been reviewed and accepted by the student's advisor, on behalf of the advisory committee, and by the Director of Graduate Studies (DGS), on behalf of the program; we verify that this is the final, approved version of the student's thesis including all changes required by the advisory committee. The undersigned agree to abide by the statements above.

Connor R. Ferguson, Student

Dr. Jonathan F. Wenk, Major Professor

Dr. Alexandre Martin, Director of Graduate Studies 


\title{
QUANTIFICATION OF PAPILLARY MUSCLE MOTION AND MITRAL REGURGITATION AFTER MYOCARDIAL INFARCTION
}

\section{$\longrightarrow$ \\ THESIS \\ A thesis submitted in partial fulfillment of the} requirements for the degree of Master of Science in Mechanical Engineering in the

College of Engineering at the University of Kentucky

\author{
By \\ Connor Randolph Ferguson \\ Lexington, Kentucky \\ Director: Dr. Jonathan F Wenk, Professor of Mechanical Engineering \\ Lexington, Kentucky \\ 2019
}

Copyright (C) Connor Randolph Ferguson 2019

https://orcid.org/0000-0003-2489-2237 


\section{ABSTRACT OF THESIS}

\section{QUANTIFICATION OF PAPILLARY MUSCLE MOTION AND MITRAL REGURGITATION AFTER MYOCARDIAL INFARCTION}

Change in papillary muscle motion as a result of left ventricular (LV) remodeling after posterolateral myocardial infarction is thought to contribute to ischemic mitral regurgitation. A finite element (FE) model of the LV was created from magnetic resonance images acquired immediately before myocardial infarction and 8 weeks later in a cohort of 12 sheep. Severity of mitral regurgitation was rated by two-dimensional echocardiography and regurgitant volume was estimated using MRI. Of the cohort, 6 animals (DC) received hydrogel injection therapy shown to limit ventricular remodeling after myocardial infarction while the control group (MI) received a similar pattern of saline injections. LV pressure was determined by direct invasive measurement and volume was estimated from MRI. FE models of the LV for each animal included both healthy and infarct tissue regions as well as a simulated hydrogel injection pattern for the DC group. Constitutive model material parameters for each region in the FE model were assigned based on results from previous research. Invasive LV pressure measurements at end diastole and end systole were used as boundary conditions to drive model simulations for each animal. Passive stiffness (C) and active material parameter (Tmax) were adjusted to match MRI estimations of LV volume at end systole and end diastole. Nodal positions of the chordae tendineae (CT) were determined by measurements obtained from the excised heart of each animal at the terminal timepoint. Changes in CT nodal displacements between end systole and end diastole at 0 and 8-week timepoints were used to investigate the potential contribution of changes in papillary muscle motion to the progression of ischemic mitral regurgitation after myocardial infarction. Nodal displacements were broken down into radial, circumferential, and longitudinal components relative to the anatomy of the individual animal model. Model results highlighted an outward radial movement in the infarct region after 8 weeks in untreated animals, while radial direction of motion observed in the treated animal group was preserved relative to baseline. Circumferential displacement decreased in the remote region in the untreated animal group after 8 weeks but was preserved relative to baseline in the treated animal group. MRI estimates of regurgitant volume increased significantly 
in the untreated animal group after 8 weeks but did not increase in the treated group. The results of this analysis suggest that hydrogel injection treatment may serve to limit changes in papillary muscle motion and severity of mitral regurgitation after posterolateral myocardial infarction.

KEYWORDS: Magnetic Resonance Imaging, Finite Element Modeling, Displacement, Volume Analysis, Mitral Regurgitation

Connor Randolph Ferguson

(Name of Student)

$04 / 18 / 2019$

Date 
QUANTIFICATION OF PAPILLARY MUSCLE MOTION AND MITRAL REGURGITATION AFTER MYOCARDIAL INFARCTION

By

Connor Randolph Ferguson

Jonathan F Wenk

Director of Thesis

Alexandre Martin

Director of Graduate Studies

$04 / 18 / 2019$

Date 


\section{DEDICATION}

To Abigail, Finley, Tom, Judy, Colin, and Fio. 


\section{ACKNOWLEDGMENTS}

The work presented here would not have been possible without the guidance of Dr. Jonathan Wenk. Dr. Wenk's knowledge and mastery of the fundamental engineering principles presented in this thesis are truly inspirational. Perhaps the best testament to this capacity lies in Dr. Wenk's eagerness and ability to communicate the complex principles of continuum mechanics and the finite element method in such an effective, easy to understand, and approachable manner. I thank Dr. Wenk for his patience, time, effort, many meetings, and in-depth discussions of mechanics and physiology. I thank Dr. Kenneth Campbell for his support, insight, and discussions of physiology and statistics throughout the course of this project, as well as his input on this thesis as part of the thesis committee. Finally, I would like to thank Dr. Hailong Chen for his time and input as part of the thesis committee.

I would like to thank the members of the computational biomechanics lab for their help and support throughout this journey. Thank you to Kurtis Mann for sharing most of this journey with me, and for being there to help each other refine our research. Thank you to Zhanqiu Liu for your help in so many ways, and your dedication to effectively and thoroughly teaching the methods of your research to your lab mates. Thank you to Amir, Hossein, and Dara for making the shared lab space a welcoming and enjoyable place to work. 


\section{TABLE OF CONTENTS}

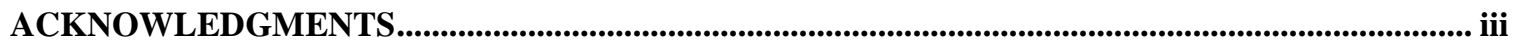

LIST OF TABLES _.........................................................................................................................................

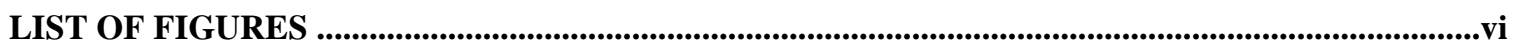

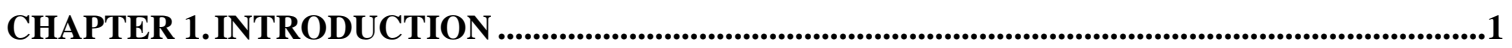

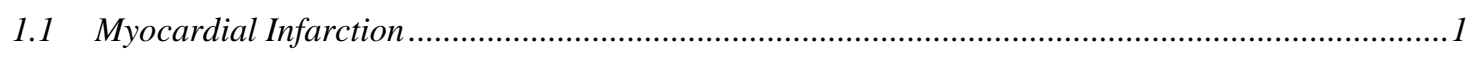

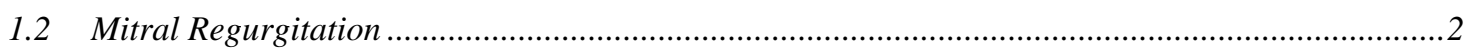

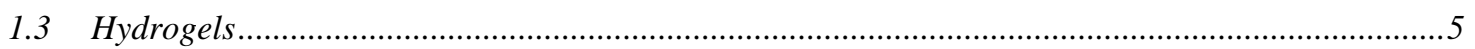

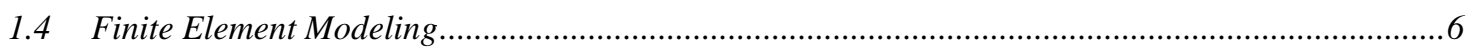

CHAPTER 2.ANALYSIS ...............................................................................................................................

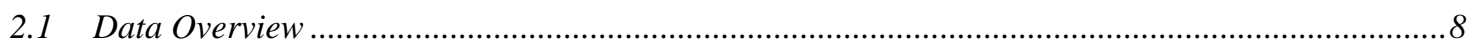

$2.2 \quad$ Finite Element Model...................................................................................................... 11

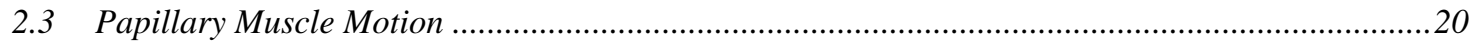

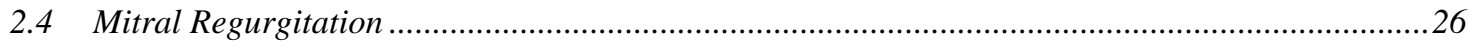

CHAPTER 3. RESULTS.........................................................................................................................29

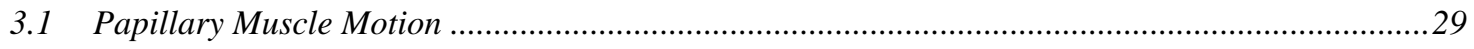

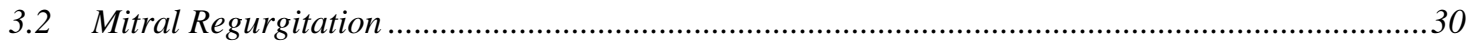

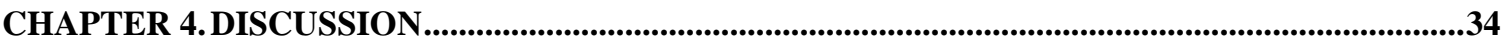

CHAPTER 5. CONCLUSION .....................................................................................................................38

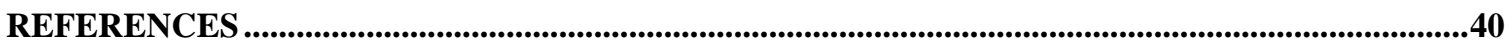

VITA 


\section{LIST OF TABLES}

Table 2.1 Comparison of constitutive model parameters between the MI and DC group in the remote and infarct regions at 8 -weeks post-MI. Data are presented as mean \pm

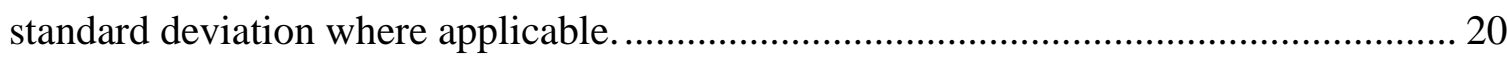

Table 3.1 Average left ventricle papillary muscle displacement in the remote tissue region. Data are presented as $\mathrm{mm} \pm$ standard deviation.

Table 3.2 Average left ventricle papillary muscle displacement in the infarct tissue region. Data are presented as $\mathrm{mm} \pm$ standard deviation.

Table 3.3 Comparison between MRI estimates of regurgitant volume, regurgitant fraction, and rating by two-dimensional echocardiography. Data are presented as mean \pm standard deviation. 31

Table 3.4 Comparison of regurgitant volume and regurgitant fraction between treatment groups. Data are presented as mean \pm standard deviation.

Table 3.5 Comparison of average mid-LV diameter at end diastole and end systole between groups and timepoints. Data are presented as mean \pm standard deviation. 33 


\section{LIST OF FIGURES}

Figure 1.1 Normal flow of blood through the heart involves filling the left ventricle with oxygenated blood from the left atrium during diastole. Contraction of the ventricle during systole causes blood to be pumped out of the left ventricle through the aorta to the rest of the body. Figure 1.1 is reproduced from Martini et al [4] .................................... 3

Figure 2.1 Unwrapped left ventricular site map showing ligated obtuse marginal branches (OM2), ligation site (orange $\mathrm{X}$ ), infarct outline markers (green $\mathrm{O}$ ), and injection sites (red

$\mathrm{X})$. 9

Figure 2.2 Hydrogel injections can be seen in this cross section of infarcted myocardium in a DC treatment group excised heart at the 8 week terminal timepoint. 10

Figure 2.3 LV endocardial contours on one short axis slice (a) immediately before infarct and (b) 8 weeks later in the same untreated animal. 11

Figure 2.4 Left ventricular endocardial contours assembled in 3D space in MATLAB. . 12 Figure 2.5 Left ventricular endocardial and epicardial IGES surfaces generated from stack contours and rendered in Creo Parametric 4.0 (PTC, Inc., Boston, MA)................ 13 Figure 2.6 Full LV FE model (a) and FE model short axis slice (b) immediately before infarct (1) and 8 weeks later (2) in the same untreated animal. Red elements represent healthy myocardium while blue elements represent the infarcted myocardium region. .. 14 Figure 2.7 MRI reconstruction of hydrogel injection pattern after initial injection in one representative animal. This figure is reproduced from Wang et al [33]. 
Figure 2.8 Representative DC group animal left ventricle model at 8 weeks showing healthy myocardium (red), infarct tissue region (blue) and injected hydrogel material in the mid wall region (green). 16

Figure 2.9 Experimentally measured length dependent active tension at different concentrations of external calcium (a) and time and length dependent active force generation (b) in cardiac myocytes compared to model estimations (dashed) [13, 14]... 19 Figure 2.10 Unwrapped left ventricle from an excised heart highlighting the infarct tissue region (blue), papillary muscles (green), and chordae tendineae attachment site (red)... 21 Figure 2.11 Three-dimensional node location (blue), CT node displacement vectors (orange) from end diastole to end systole, and LV long axis (green) calculated in MATLAB for the same animal at 0 week (a) and 8 week (b) timepoints. Global z axis is shown for both end diastolic and end systolic figures but does not apply to the relative motion figure

Figure 2.12 End diastolic LV endocardial nodes (dark blue) for one model highlighting CT attachment node locations (light blue), calculated long axis of the ventricle (green), CT attachment node motion vectors (orange) and local coordinate system for one of the CT attachment nodes (red).

Figure 2.13 Closeup view of a small portion of the LV endocardial node cloud (dark blue) highlighting papillary CT attachment nodes (light blue), motion vectors (orange), and local coordinate system for one CT attachment node (red). 25

Figure 2.14 Two-dimensional echo apical view of a sheep heart after myocardial infarction showing (a) the left ventricle (LV), left atrium (LA), and aorta LV outflow tract (Ao); (b) color Doppler overlay of the same view showing an MR rating of $1+\ldots . .26$ 
Figure 2.15 Two-dimensional echo apical view of a sheep heart after myocardial infarction showing (a) the left ventricle (LV), left atrium (LA), and aorta LV outflow tract (Ao); (b) color Dopper overlay of the same view showing an MR rating of $3+\ldots . . .27$ 


\section{CHAPTER 1. INTRODUCTION}

\subsection{Myocardial Infarction}

The term myocardial infarction (MI) is generally used to denote the cell death of cardiac myocytes as a result of ischemia, the restriction in blood supply to tissue that leads to a shortage of oxygen needed for cellular metabolism. Non-ischemic cardiomyopathy or other inflammatory diseases can also lead to loss of functional myocardium. According

to Thygesen et al., approximately one-third of the world population will die as a result of cardiovascular disease, and the largest portion of these deaths will occur in cases involving some form of myocardial infarction [31]. The pathophysiology of left ventricular (LV) remodeling after MI is well documented and is primarily characterized by structural and functional changes.

Degradation or absolute elimination of contractile function in infarcted tissue regions due to myocyte death can cause abnormal loading and stress conditions within the myocardium. These conditions can lead to decreased cardiac output and initiate biomechanical and biochemical compensatory reparative mechanisms which can result in dilation, hypertrophy, and scarring. Early remodeling, which may occur within hours to days of an adverse event, is generally localized to thinning and expansion of the infarcted tissue region due to degradation of myocyte collagen integrity, while late remodeling is characterized by global LV dilation, distortion, and deterioration of contractile function which can continue over weeks and months following MI. Inability to adequately compensate for increases in myocardium wall stress can eventually lead to a cascade of progressive dilation and cell death as cells in the border zone myocardium surrounding the infarcted tissue region are incorporated into the infarct [30]. 
Due to the prevalence of myocardial infarction in cardiovascular disease, treatment for regulating LV remodeling after MI has been investigated in depth. Traditional treatment methods to prevent LV remodeling have focused on infarct reperfusion and pharmacological intervention; however, mechanical surgical countermeasures that focus on physical reinforcement of the infarcted tissue region via suture rows, ventricle wraps, balloon inflation, and structural patches have been shown to be effective in limiting LV remodeling [21]. Expansion of stem cell research has shown that the application of stem cell differentiation can be used as an effective treatment to regenerate infarcted myocardium. A 2001 animal study by Orlic et al. reported a 68\% occupation of newly formed myocardium within the infarcted portion of the ventricle 9 days after transplantation of bone marrows cells in mice [26]. More recent research has focused on the implementation of injectable hydrogels both for their efficacy as a vehicle for introduction of regenerative stem cells into the myocardium and for structural reinforcement due to highly tunable mechanical properties.

\subsection{Mitral Regurgitation}

Myocardial infarction (MI) is known to cause left ventricular (LV) remodeling associated with dilatation and distortion of the ventricular shape leading to deterioration in contractile function [30]. The normal path of blood through the left side of the heart as shown in Figure 1.1 involves filling of the LV with oxygenated blood from the left atrium during diastole which is then ejected through the aorta to the rest of the body when the heart contracts during systole. 
(g) One-way flow through the heart is ensured by two sets of valves.

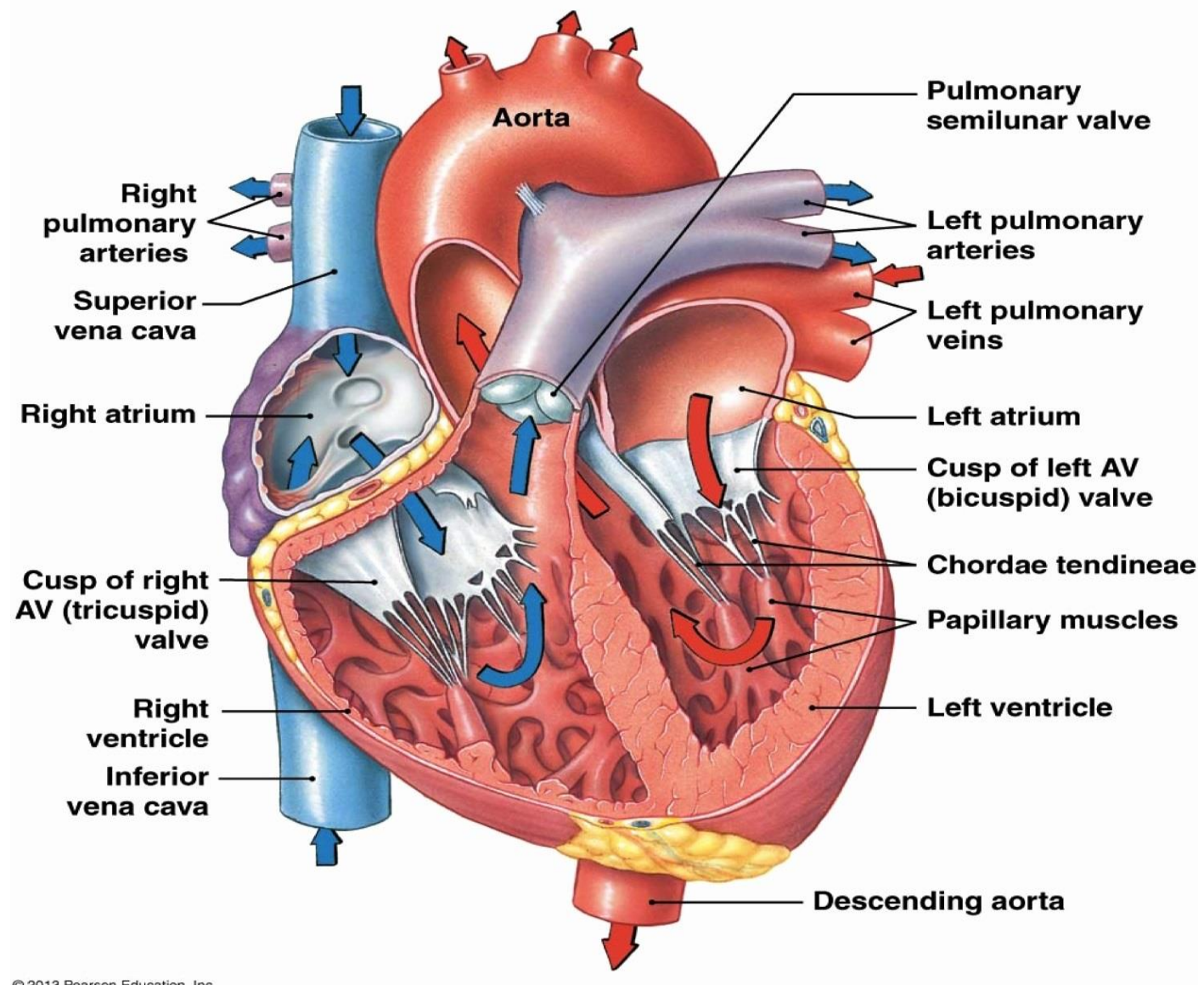

Figure 1.1 Normal flow of blood through the heart involves filling the left ventricle with oxygenated blood from the left atrium during diastole. Contraction of the ventricle during systole causes blood to be pumped out of the left ventricle through the aorta to the rest of the body. Figure 1.1 is reproduced from Martini et al [4].

The mitral valve operates as a one-way valve to allow blood to flow into the LV during diastole while preventing flow in the opposite direction. Improper closure of the mitral valve can result in an adverse condition known as mitral regurgitation in which blood flows backward from the LV to the left atrium. A previous population-based study by Bursi et 
al. identified mitral regurgitation in as many as $50 \%$ of patients after the first myocardial infarction [3]. The presence of mitral regurgitation was found to be an independent predictor of cardiovascular mortality in post-MI patients [20]. Due to tethering between the LV papillary muscles and the mitral valve via the chordae tendineae, papillary muscle dysfunction was clinically assumed to be associated with mitral regurgitation despite the lack of objective evidence.

Echocardiographic examination of the mitral leaflets and left ventricular myocardium was used in early studies to investigate the role of the papillary muscles in mitral valve closure [10,22]. Results from Godley et al. provide evidence for development of mitral regurgitation from dyskinesis of the LV myocardium involving the papillary muscles [22]. A more recent study by Jensen et al. assessed the role of papillary muscle displacement in a porcine model of ischemic mitral regurgitation using 3-dimensional morphology cardiac MRI [17]. Jensen et al. reported the distance between the anterior and posterior papillary muscle to be a predictor of LV regurgitant volume but did not assess changes in distances during the cardiac cycle. In addition to papillary muscle displacement and overall LV dilation, change in papillary muscle motion throughout the cardiac cycle as a result of left ventricular remodeling after posterolateral myocardial infarction may contribute to ischemic mitral regurgitation [35].

This group previously showed that in-vivo injected shear-thinning biomaterial significantly improved regional contractile function in the border zone and reduced LV remodeling in a cohort of Dorset sheep when compared to untreated controls [33]. The present study uses a similar animal-specific MRI-based FE model to quantify papillary muscle motion before and 8 weeks after MI in the same ovine cohort. This approach 
allows us to investigate the potential contribution of changes in papillary muscle motion to the progression of ischemic mitral regurgitation after myocardial infarction.

\subsection{Hydrogels}

Synthetic hydrogels have been developed and implemented in a wide range of applications since their creation in the 1950s by Wichterle et al [1]. A hydrogel consists of a network of polymer chains with functional crosslinking synthesized from hydrophilic monomers. Due to the nature of their composition, a hydrogel can swell to accommodate large quantities of water which allows for flexibility similar to natural biological tissue. Furthermore, biocompatible hydrogels have been developed using naturally occurring extracellular matrix components such as hyaluronic acid. The mechanism of cross linking as well as the type of polymer chain being crosslinked affects the properties of the hydrogel and allows for the introduction of a diverse range of desirable attributes depending on the application. As briefly discussed in a previous section of this document, recent research has investigated several applications of hydrogels in the medical field such as absorbable sutures, tissue engineering and regeneration, and drug delivery $[1,9]$. This thesis will focus on the use of an injectable biological hydrogel treatment shown to enhance the mechanical properties of an infarcted tissue region and limit left ventricular remodeling following myocardial infarction. 


\subsection{Finite Element Modeling}

Modelling cardiac mechanical properties in vivo has been a challenging problem for researchers. While traditional in vitro material property testing methods can provide useful information about the behavior of cardiac tissue, functional forms and parameters of the governing constitutive equations used in modeling must describe material properties for both resting and active, as well as normal and diseased myocardium, in vivo. One of the most challenging aspects of cardiac tissue modeling is that the constitutive properties of myocardium are three dimensional, anisotropic, nonlinear, and time dependent [5], properties that were shown previously via in vitro biaxial tests conducted by Yin and Demer in 1983 [38].

In order to account for the myofiber structure of the myocardium, Guccione et al. developed a transversely isotropic exponential strain function which includes a system of fiber oriented strain components [15]. More recent work by Wenk et al. has expanded on the Guccione methods to incorporate and investigate regional contractility and stress differences within a left ventricular finite element model [36, 37]. The capability to incorporate regional inhomogeneity of material properties into the cardiac model has enabled the investigation of contractility and stress within the myocardium of a ventricle that has been compromised by myocardial infarction. Wenk et al. used these methods to develop a ventricle model with healthy, infarct, and border zone tissue regions in order to objectively quantify and characterize stress distributions within the border zone region that potentially drive the recruitment of border zone myocytes into the infarcted tissue region discussed in an earlier section [37]. In 2009, Wenk et al. published their methods of simulation and optimization of polymeric injection patterns within a diseased ventricle to 
reduce adverse myocardium wall stress and limit ventricular remodeling. Furthermore, Wenk et al. provided insights into the development of ischemic mitral regurgitation using the first finite element model of the full left ventricle with mitral valve [35].

More recently, Wang et al. used the Guccione methods previously described to investigate transmural differences in left ventricular contractility [34]. The same group, in conjunction with the Gorman Cardiovascular Group at the University of Pennsylvania, developed and implemented animal specific finite element models of the left ventricle with healthy and infarcted tissue regions as well as a novel hydrogel injection treatment shown to limit LV remodeling in order to estimate regional myocardial stress and contractility in a cohort of sheep [33, 27]. The work discussed in this thesis uses these methods to assess changes in papillary muscle motion and mitral regurgitation following myocardial infarction in the same cohort of animals. Data collection and analysis methods are discussed in greater detail in chapter two of this thesis. Results of the analysis and discussion of the work presented in this thesis are reported in chapters three and four respectively. Finally, chapter five provides a conclusion of this work as well as prospects for future study and application. 


\section{CHAPTER 2. ANALYSIS}

\subsection{Data Overview}

The data presented in this study were previously collected as part of a study by Rodell et al. [27] in compliance with the University of Pennsylvania's Institutional Animal Care and Use Committee and in agreement with the National Institute of Health's guidelines for the care and use of laboratory animals (NIH Publication 85-23, revised 1996). In summary, a cohort of Dorset sheep underwent a left thoracotomy and subsequent posterolateral infarction produced by suture ligation of obtuse marginal branches to result in an infarct area of approximately $20 \%$ of the LV and involving the posterior papillary. Infarct area coverage of the papillary muscle was unsatisfactory in one animal from each of the untreated and treated groups, and those data are excluded from this analysis. Thirty minutes post ligation, 16 injections $(0.3 \mathrm{~mL}$ each via $1 / 2 \mathrm{~mL}$ syringe, with $27 \mathrm{G} 1 / 2$ " needle) consisting of saline (MI control) or hydrogel (DC treatment) were administered via direct injections to the infarct region during thoracotomy. As a proof of concept, the Gorman group demonstrated successful administration of hydrogel through percutaneous intramyocardial injection to the inferior and anterior walls of the LV via the right carotid as well as to the RV septal wall via the internal jugular.

The shear-thinning, self-healing hydrogel treatment investigated in this study is a modified hyaluronic acid polymer entirely designed and implemented by Rodell et al. The hydrogel used in this study includes a secondary crosslinking of polymer chains, also referred to as dual-crosslinking (DC) to enhance mechanical properties and prolong degradation. A representative ligation site, infarct area, and injection location map for one animal in the treated DC group are shown in Figure 2.1. 


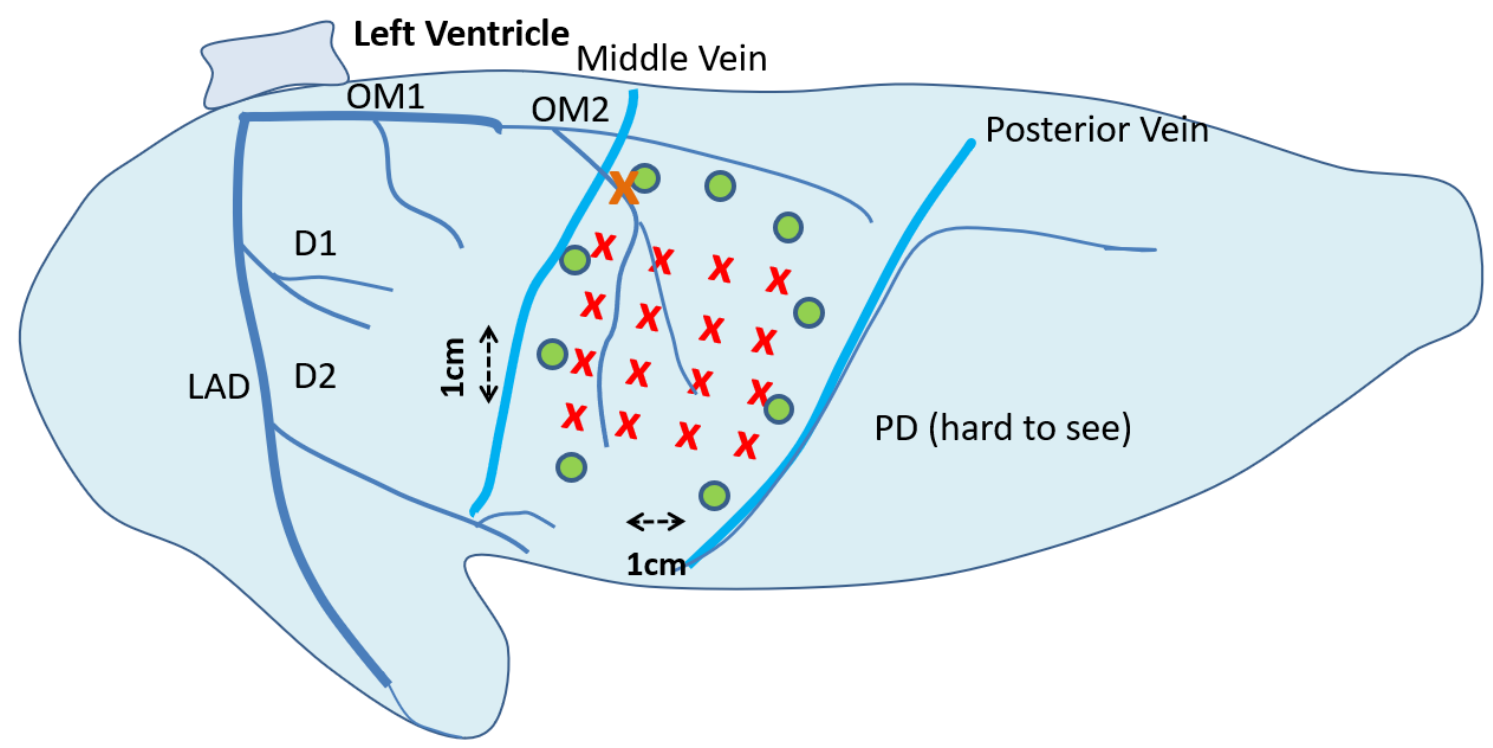

Figure 2.1 Unwrapped left ventricular site map showing ligated obtuse marginal branches (OM2), ligation site (orange $\mathrm{X}$ ), infarct outline markers (green $\mathrm{O}$ ), and injection sites (red $\mathrm{X})$.

Intramyocardial hydrogel injections can be seen in the cross section of the infarct tissue region of an excised heart of a DC treatment group animal at the 8 week timepoint in Figure 2.2 . 


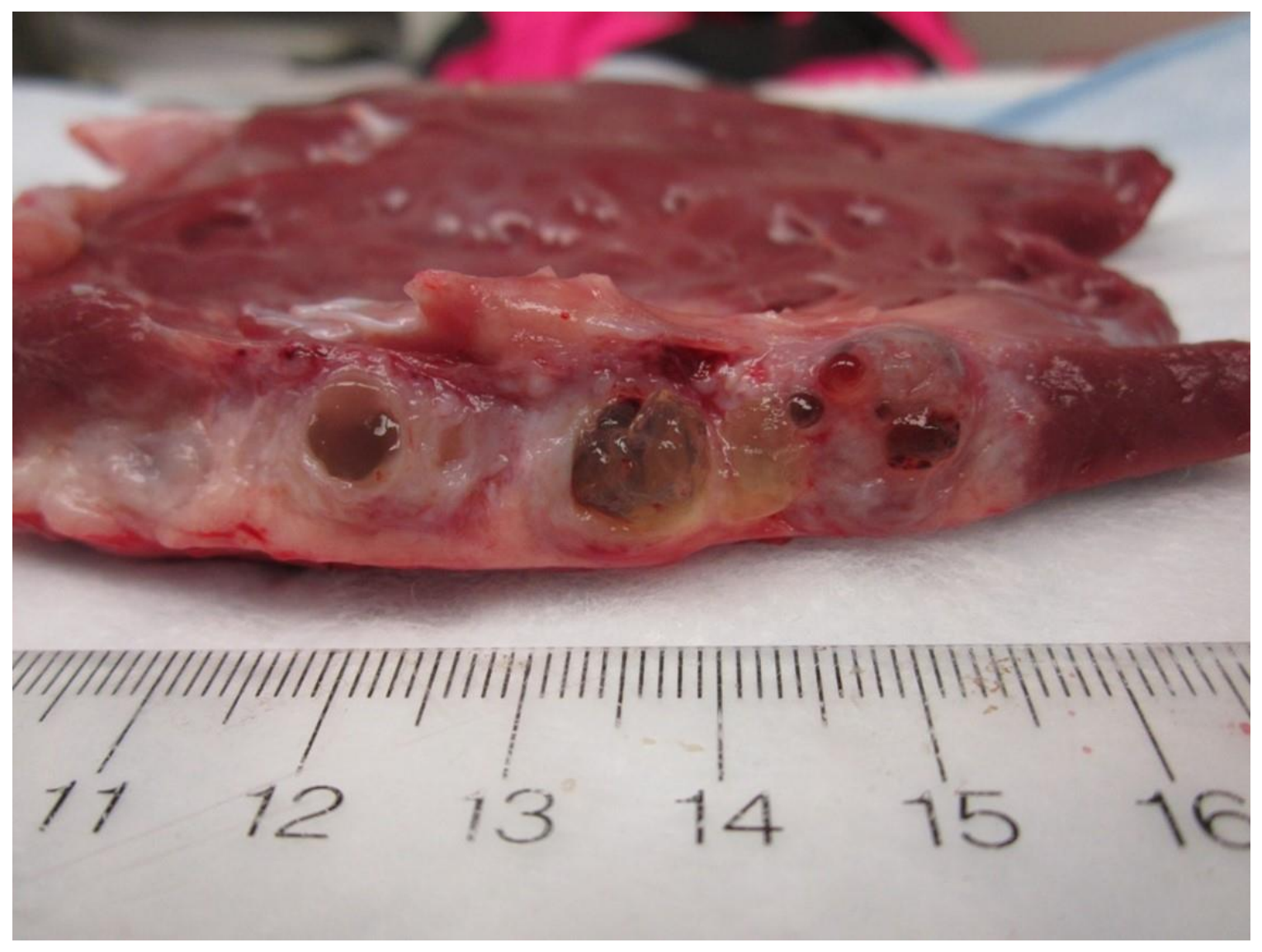

Figure 2.2 Hydrogel injections can be seen in this cross section of infarcted myocardium in a DC treatment group excised heart at the 8 week terminal timepoint.

MRI acquisition was performed at 3T (Tim Magnetom Trio Scanner; Siemens, Inc.) immediately before infarct as well as 8 weeks post infarct. Cardiac gating was performed by placement of a pressure catheter (Millar Instruments, Inc.) into the LV. 2D cine MRI scans were conducted with the following parameters: field of view $=280 \times 166.25 \mathrm{~mm}$, acquisition matrix $=256 \times 152$ pixels, repetition time $=27.52 \mathrm{~ms}$, echo time $=1.46 \mathrm{~ms}, \mathrm{BW}$ $=930 \mathrm{~Hz} /$ pixel, slice thickness $=4 \mathrm{~mm}$. Infarct location and geometry was confirmed using additional late gadolinium enhancement imaging. Severity of mitral regurgitation was 
rated (scale $0-3$ ) by two-dimensional echocardiography. At 8 weeks post-MI, the animals were euthanized and the hearts were excised.

\subsection{Finite Element Model}

Left ventricular geometry in each animal at both timepoints was obtained from 2D cine image contours using ImageJ. Of importance to note is that contours included both papillary muscles as shown in Figure 2.3, which is different than certain types of image analysis.

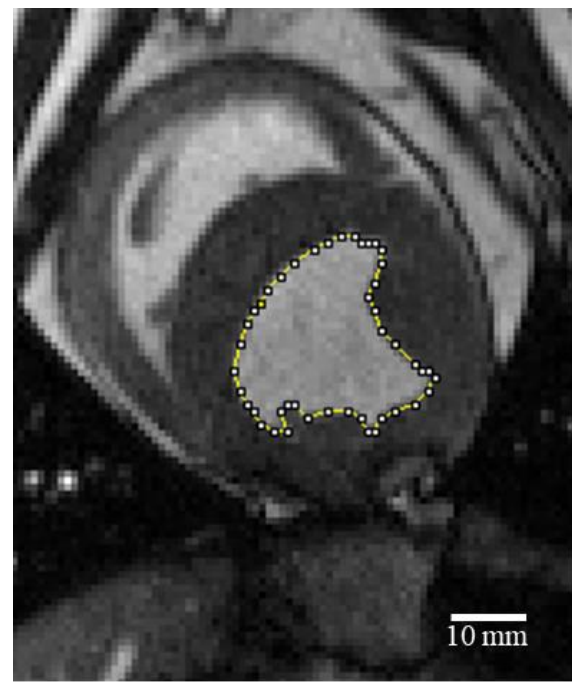

(a)

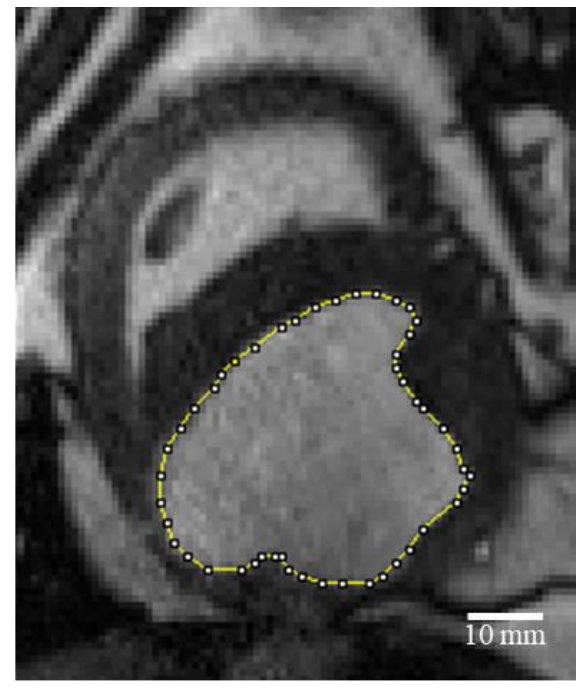

(b)

Figure 2.3 LV endocardial contours on one short axis slice (a) immediately before infarct and (b) 8 weeks later in the same untreated animal.

A custom MATLAB (MathWorks, Inc., Natick, MA) script was developed to process the contours from Image $\mathbf{J}$ by importing and converting contour point locations for each slice from voxel dimensions to millimeters. Left ventricular contours can be assembled into a 
vertical stack to represent the ventricle in three-dimensional space as shown in Figure 2.4 .

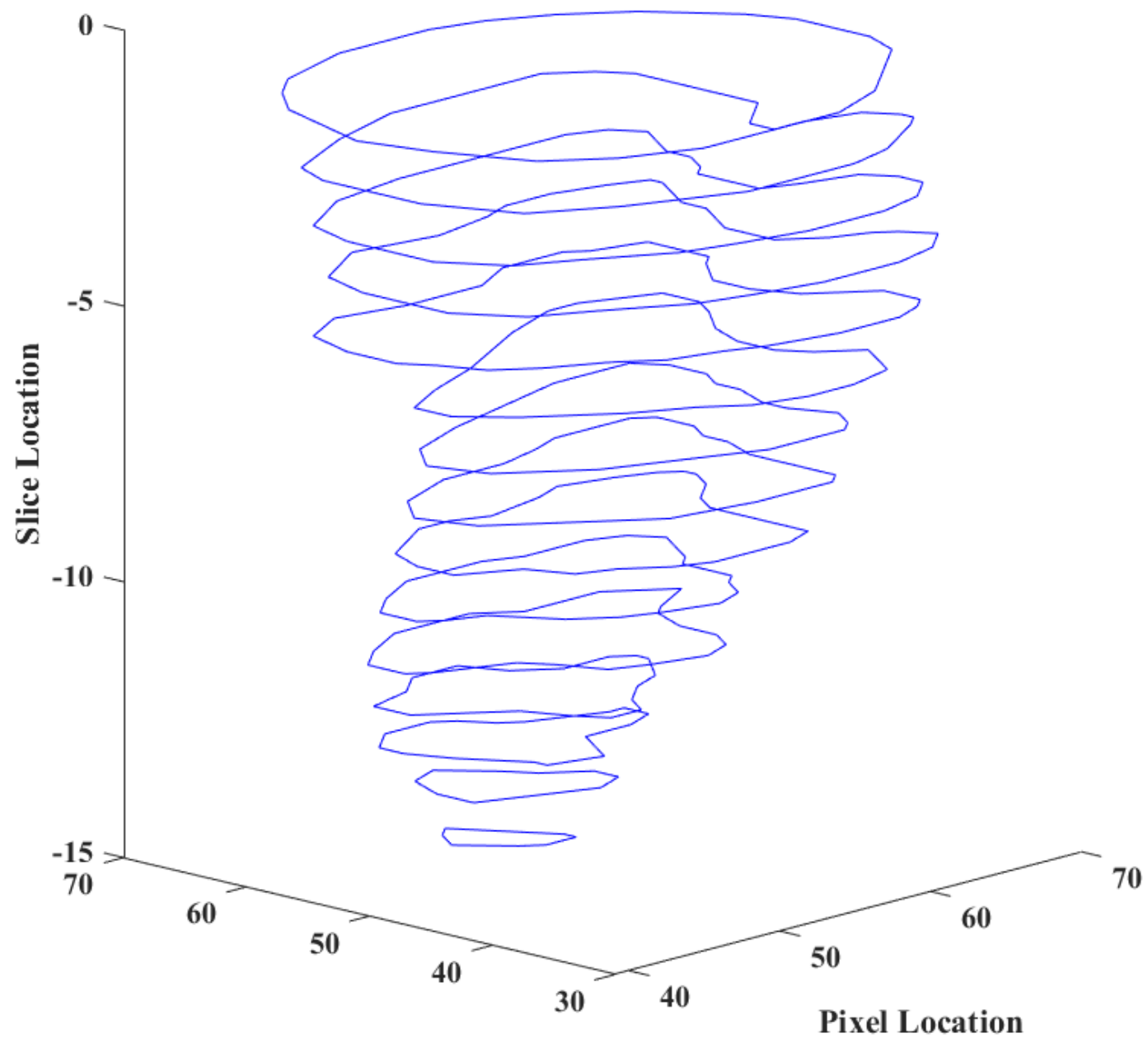

Figure 2.4 Left ventricular endocardial contours assembled in 3D space in MATLAB. 
Surfaces were generated from endocardial and epicardial contours as shown in Figure 2.5. This was accomplished by fitting the contour points with geometric surfaces using the software Rapidform (INUS Technology, Inc, Sunnyvale, CA).

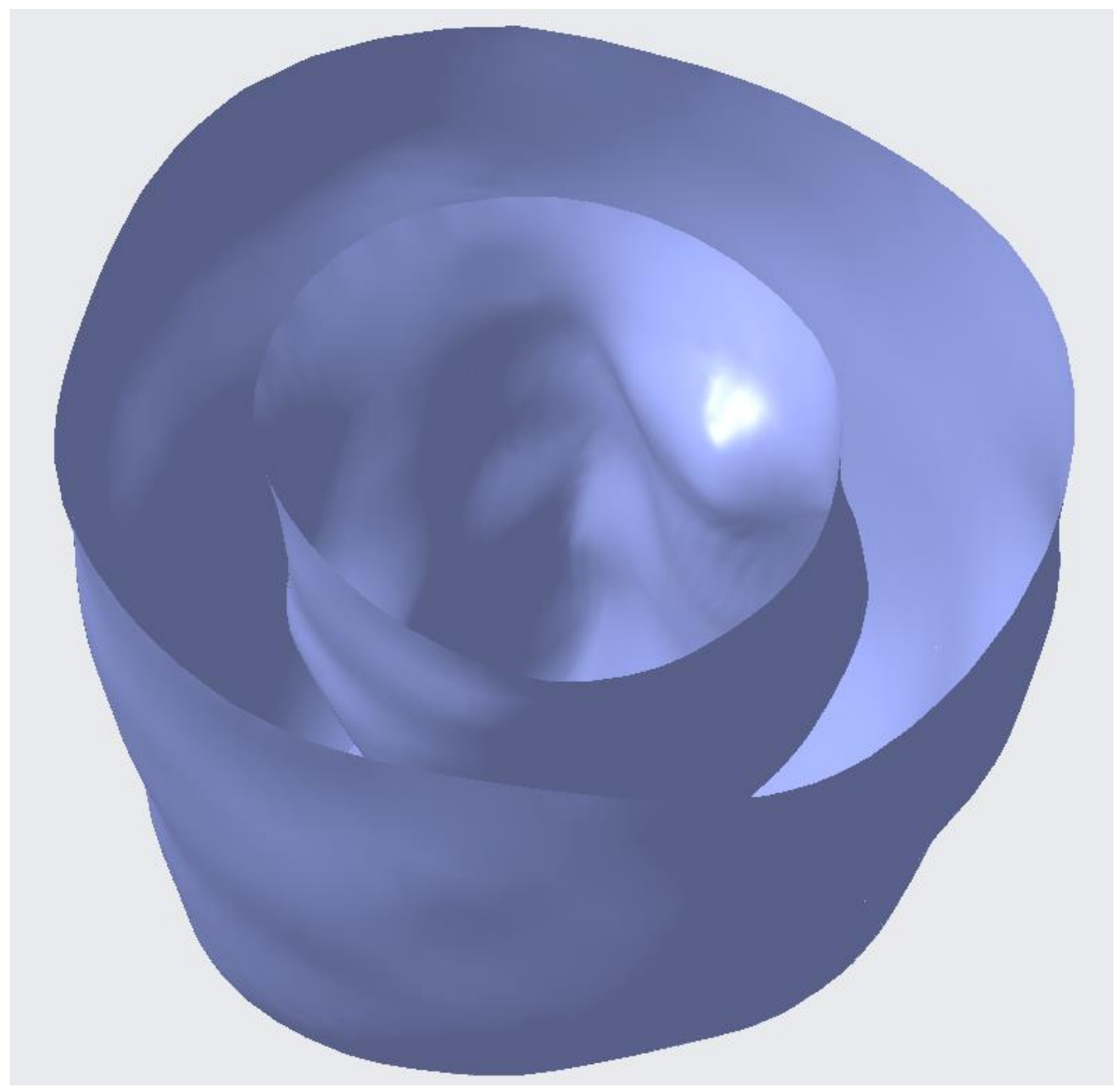

Figure 2.5 Left ventricular endocardial and epicardial IGES surfaces generated from stack contours and rendered in Creo Parametric 4.0 (PTC, Inc., Boston, MA).

The IGES surfaces generated from the endocardial and epicardial contour slices were then used to create individual finite element (FE) models for each animal at the 0 week and 8 week timepoint using trilinear 8-node hexahedral brick elements (TrueGrid, XYZ 
Scientific, Inc.). The LV wall was divided evenly into three transmural layers from endocardium to epicardium as shown in Figure 2.6.

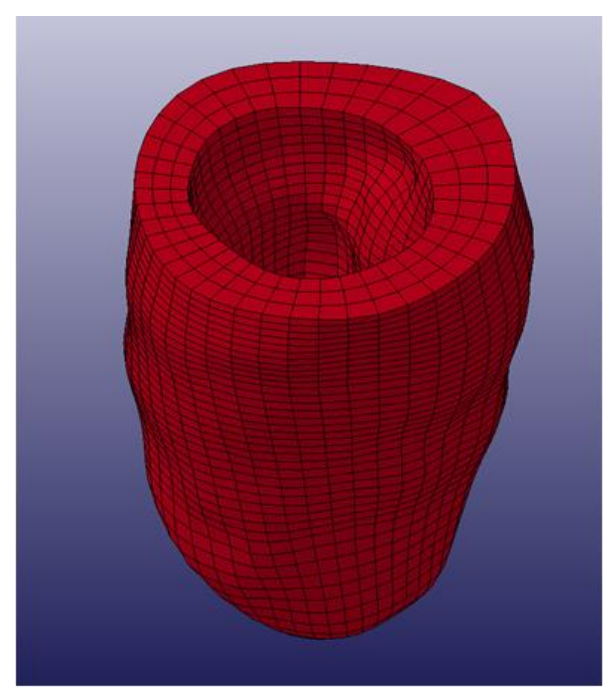

(1a)

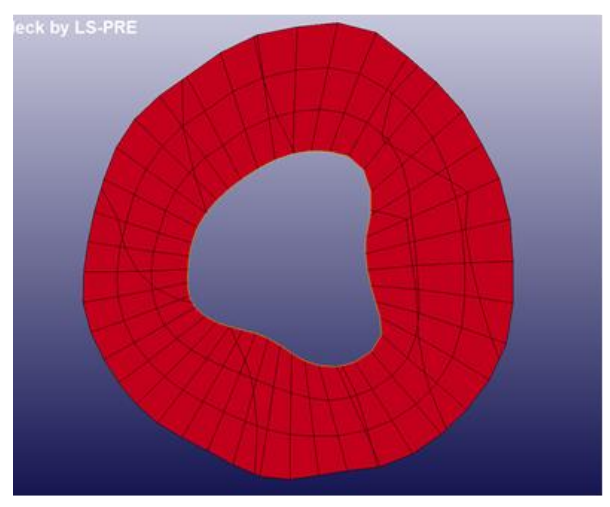

(1b)

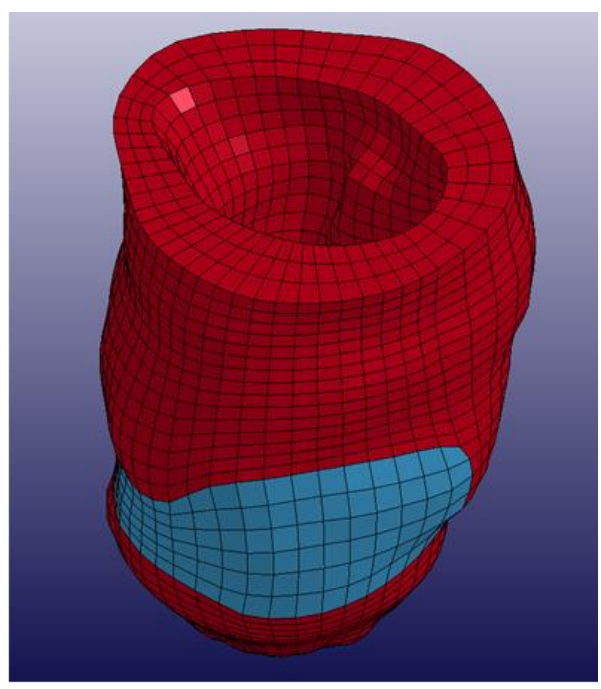

(2a)

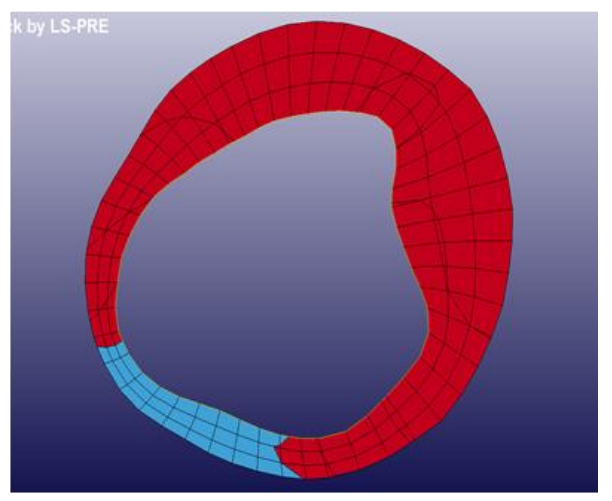

(2b)

Figure 2.6 Full LV FE model (a) and FE model short axis slice (b) immediately before infarct (1) and 8 weeks later (2) in the same untreated animal. Red elements represent healthy myocardium while blue elements represent the infarcted myocardium region.

FE models for each animal at the 8 week timepoint included both healthy and infarcted tissue regions as well as a simulated hydrogel injection pattern for the treated DC group 
based on previous MRI data [10]. MRI reconstruction of the hydrogel injection pattern observed in a representative animal after initial injection is shown in Figure 2.7.

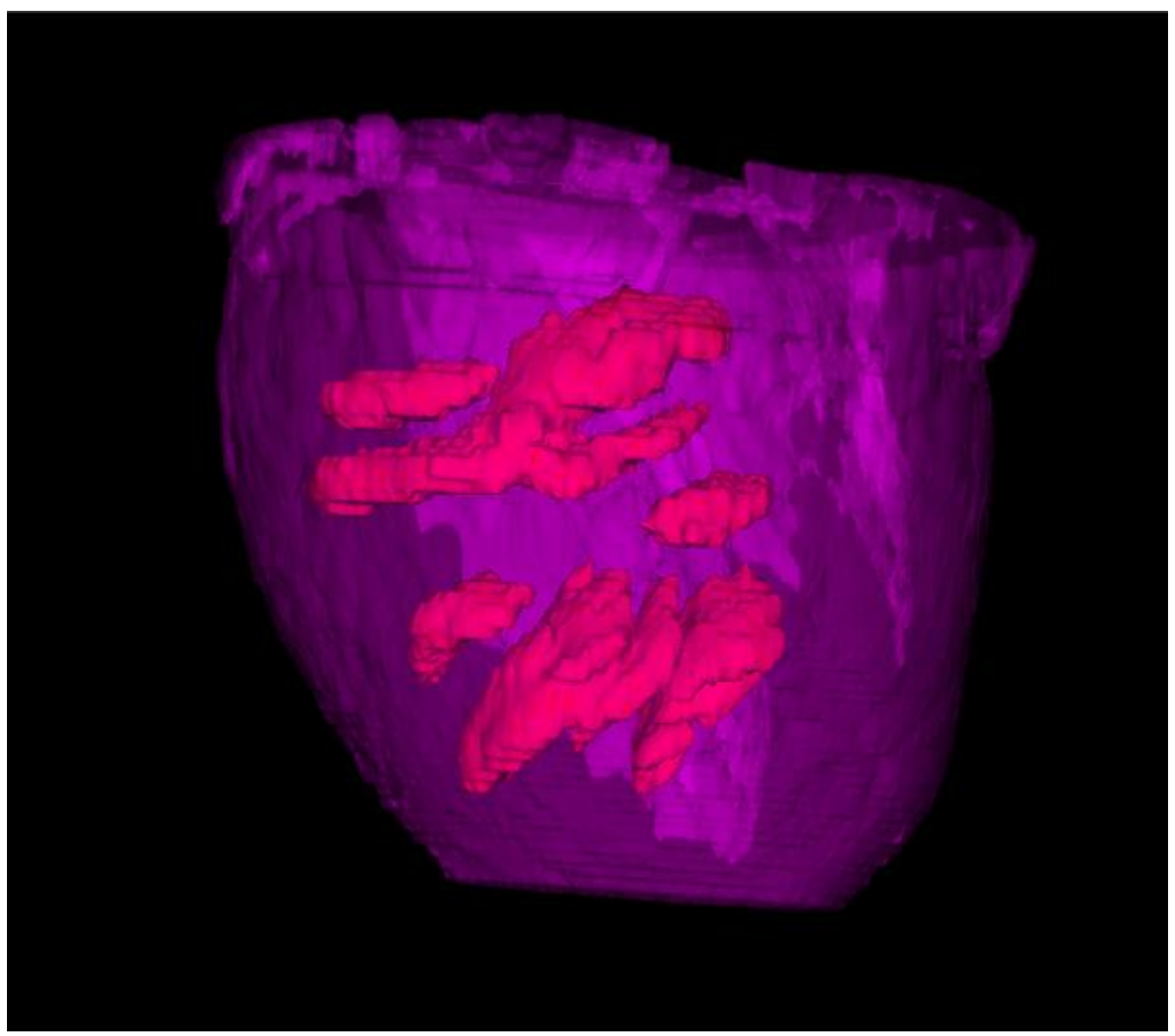

Figure 2.7 MRI reconstruction of hydrogel injection pattern after initial injection in one representative animal. This figure is reproduced from Wang et al [33].

A simulated hydrogel injection pattern was built into the mid wall of the myocardium within the infarct region of the treated group animal models using the patterns observed in the MRI reconstruction presented in Figure 2.7. A representative model with hydrogel injection pattern is shown in Figure 2.8. 


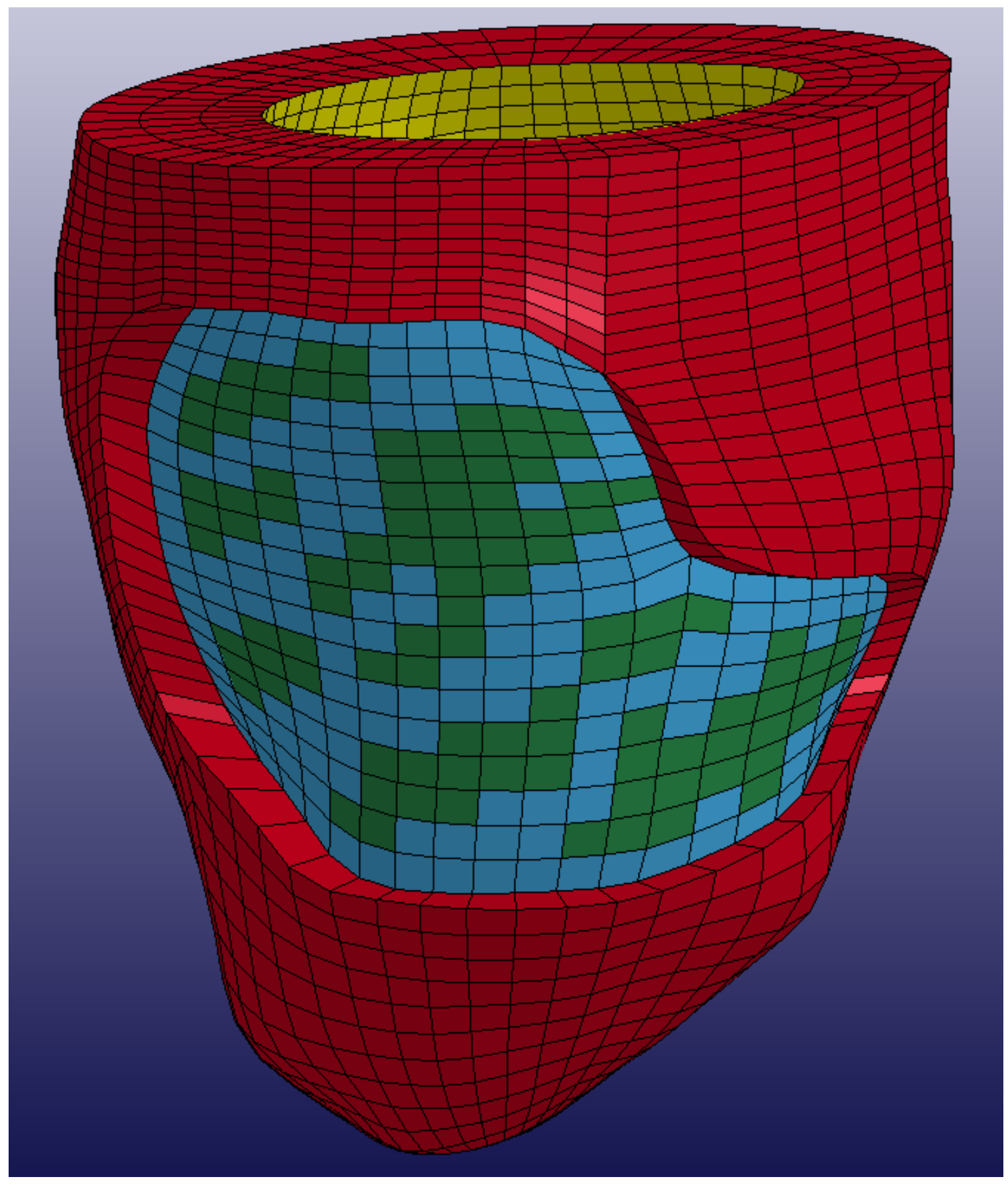

Figure 2.8 Representative DC group animal left ventricle model at 8 weeks showing healthy myocardium (red), infarct tissue region (blue) and injected hydrogel material in the mid wall region (green). 
Cardiac myofiber distribution within each element was assigned in both infarct and remote tissue regions based on the results from a previous study [6]. A myofiber distribution of $37^{\circ}$ at epicardium to $+83^{\circ}$ at endocardium, with respect to the circumferential direction, was used for the remote tissue region in both groups at all timepoints. At 8 weeks postMI, the infarct tissue region in the untreated MI group was assigned a myofiber distribution of $-18^{\circ}$ at epicardium to $+27^{\circ}$ at endocardium, while the treated DC group was assigned a myofiber distribution of $-2^{\circ}$ at epicardium to $+26^{\circ}$ at endocardium.

Each FE model simulation consisted of both a passive diastolic filling phase and an active systolic contraction phase (LS-DYNA, Livermore Software Technology Corporation). The methods used in this study to create the finite element models and the equations used to drive the model simulations were obtained from previous research [33, 37, 27]. The passive myocardium response was represented using a nearly incompressible, transversely isotropic, hyperelastic material defined by the following strain energy function $[13,14]$ :

$W_{\text {myocardium }}=\frac{C}{2} \times\left(e^{b_{f} E_{f f}^{2}+b_{t}\left(E_{S S}^{2}+E_{n n}^{2}+E_{n S}^{2}+E_{S n}^{2}\right)+b_{f s}\left(E_{f s}^{2}+E_{s f}^{2}+E_{f n}^{2}+E_{n f}^{2}\right)}-1\right)+\frac{k}{2}(J-1)^{2}$

(Eq. 2.1)

where $E_{i j}$ are the deviatoric components of the Green-Lagrange strain tensor relative to the myofiber coordinate system $(\mathrm{f}=$ fiber direction, $\mathrm{s}=$ cross-fiber in-plane direction, $\mathrm{n}=$ transverse-fiber direction) and $J$ is the determinant of the deformation gradient. The passive material parameter $\mathrm{C}$ scales stress in all directions, while $\mathrm{b}_{\mathrm{f}}$ scales the fiber direction stiffness, $b_{t}$ scales the cross-fiber direction stiffness, and $b_{\mathrm{fs}}$ scales the transverse- 
fiber direction stiffness. The response of the hydrogel injections in the DC group was represented using a nearly incompressible, isotropic, hyperelastic material law defined by the following strain energy function [33]:

$W_{\text {injection }}=\frac{E}{2(1+v)} \operatorname{tr}\left(\mathbf{E}^{2}\right)+\frac{E}{6(1-2 v)} \ln (J)^{2}$

where $\mathbf{E}$ is the deviatoric Green-Lagrange strain tensor and the material parameters for Young's modulus $(E)$ of the hydrogel were assigned based on experimental results [27]. Poisson's ratio (v) was assigned a value of 0.499 to represent near incompressibility.

Systolic stress in the myocardium, calculated as the sum of the passive stress derived from the strain energy function given in Equation 2.1 and the active stress generated along the fiber direction, is given in Equation 2.3.

$\mathbf{S}=p J \mathbf{C}^{-1}+2 J^{-2 / 3} \operatorname{Dev}\left(\frac{\partial \bar{W}}{\partial \overline{\mathbf{C}}}\right)+\mathbf{T}_{\mathbf{0}}\left\{t, C a_{0}, l, T_{\max }\right\}$

where $\operatorname{Dev}()$ is the deviatoric operator, and the active stress $\mathbf{T}_{\mathbf{0}}$ is a function of time $t$, peak intracellular calcium $\mathrm{Ca}$, sarcomere length $l$, and maximum isometric tension $T_{\max }$. Specifically, the active contraction $\mathbf{T}_{\mathbf{0}}$ is defined by the modified Hill equation given in Equation 2.4.

$\mathbf{T}_{\mathbf{0}}=\frac{1}{2} T_{\max } \frac{C a_{0}^{2}}{C a_{0}^{2}+E C a_{50}^{2}}\left(1-\cos \left(\frac{0.25}{m l_{R} \sqrt{2 E_{f f}+1}+b}+1\right) \pi\right)$ 
where $m$ and $b$ are constants, $E_{f f}$ is strain in the fiber direction, and $E C a_{50}^{2}$ is lengthdependent calcium sensitivity given in Equation 2.5.

$E C a_{50}^{2}=\frac{\left(C a_{0}\right)_{\max }}{\sqrt{\exp \left[B\left(l_{R} \sqrt{2 E_{f f}+1}-l_{0}\right)\right]-1}}$

where $B$ is a constant, $l_{0}$ is the length at which the sarcomere does not generate any force, and $l_{R}$ is the stress-free reference sarcomere length. The equations used to model length dependent active tension and external calcium concentration for cardiac myocytes were developed previously [13, 14]. Figure 2.9 shows the comparison between model estimations and experimental results for active tension.

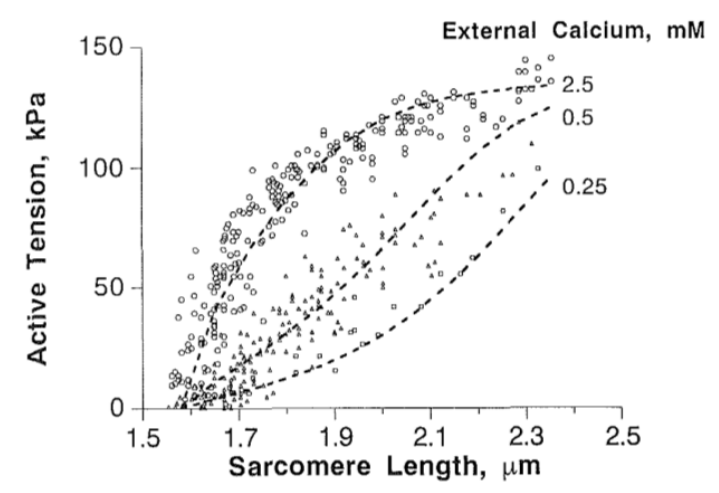

(a)

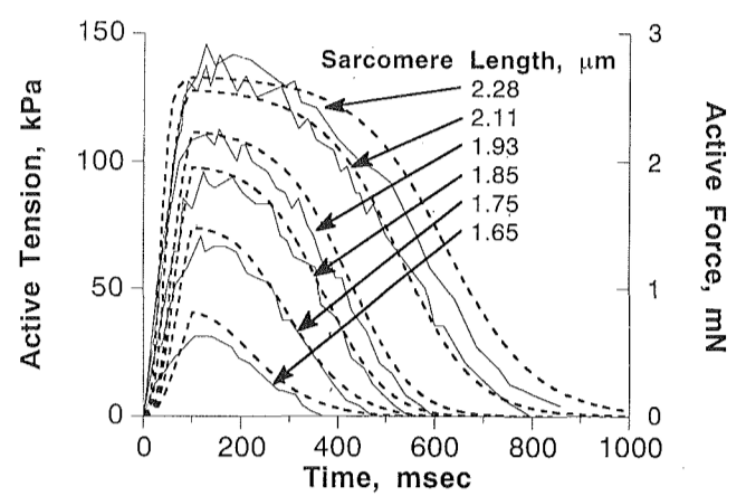

(b)

Figure 2.9 Experimentally measured length dependent active tension at different concentrations of external calcium (a) and time and length dependent active force generation (b) in cardiac myocytes compared to model estimations (dashed) [13, 14]. 
Invasive LV pressure measurements at end diastole and end systole were used as boundary conditions to drive model simulations for each animal. The passive myocardium and hydrogel material response during the diastolic filling phase were driven by the strain energy functions provided in Equations 1.1 and 1.2 respectively. Passive stiffness (C) and maximum myocyte isometric force generation $\left(\mathrm{T}_{\max }\right)$ were adjusted such that the model matched MRI LV volume estimations at end diastole and end systole. Constitutive model material parameters $\mathrm{C}, \mathrm{T}_{\max }, \mathrm{b}_{\mathrm{f}}, \mathrm{b}_{\mathrm{t}}, \mathrm{b}_{\mathrm{f}}$, for each region in the $\mathrm{FE}$ model were assigned based on results from previous studies $[3,4,5,10,13]$ and are provided in Table 2.1. Passive material parameters $\mathrm{b}_{f}, \mathrm{~b}_{t}$, and $\mathrm{b}_{f s}$ were assigned differently in the remote and infarct region based on results from previous studies [6, 24, 27, 33].

Table 2.1 Comparison of constitutive model parameters between the MI and DC group in the remote and infarct regions at 8 -weeks post-MI. Data are presented as mean \pm standard deviation where applicable.

\begin{tabular}{|c|c|c|c|c|c|c|}
\hline \multirow{3}{*}{ MI } & & $\mathrm{C}(\mathrm{kPa})$ & Tmax (kPa) & $b_{f}$ & $b_{t}$ & $\mathrm{~b}_{\mathrm{fs}}$ \\
\hline & Remote & $0.75 \pm 0.66$ & $278.7 \pm 186.4$ & 22.84 & 3.46 & 12.00 \\
\hline & Infarct & $7.41 \pm 6.92$ & - & 15.28 & 8.32 & 25.27 \\
\hline \multirow{2}{*}{ DC } & Remote & $0.09 \pm 0.06$ & $93.6 \pm 43.1$ & 22.84 & 3.46 & 12.00 \\
\hline & Infarct & $3.26 \pm 3.32$ & - & 139.26 & 18.55 & 25.74 \\
\hline
\end{tabular}

\subsection{Papillary Muscle Motion}

After completion of the simulations, full LV geometry node locations were exported at both end diastole and end systole. Nodal positions of the chordae tendineae (CT) attachment sites on the papillary muscles were determined by measurements obtained from 
the excised heart of each animal at the terminal timepoint. The left ventricle of an excised heart showing the infarct tissue region, posterior and anterior papillary muscles, and chordae tendineae attachment locations can be seen in Figure 2.10.

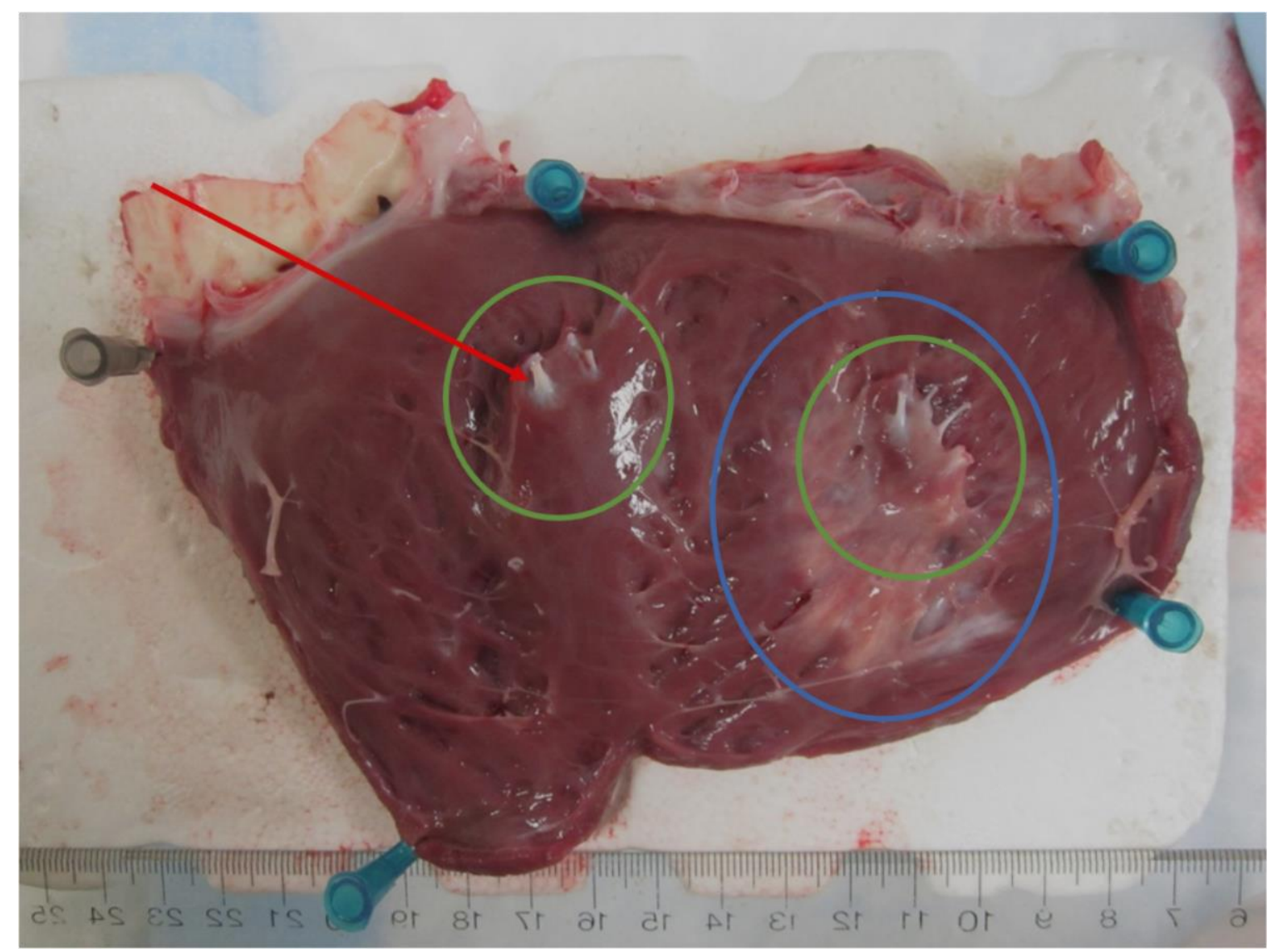

Figure 2.10 Unwrapped left ventricle from an excised heart highlighting the infarct tissue region (blue), papillary muscles (green), and chordae tendineae attachment site (red).

A custom MATLAB script was developed to process the exported node files and extract useful location and motion data of the CT attachment nodes at end diastole and end systole. A local coordinate system was created for each CT attachment node and nodal displacement from end diastole to end systole was quantified in terms of the local 
coordinate system for each corresponding node. The centroid of each ring of elements was calculated along the entire length of the ventricle, and the long axis of the ventricle was defined as the best fit line to the set of calculated centroid points and the apex node of the ventricle. Three-dimensional node and displacement vector plots are shown in Figure 2.11.

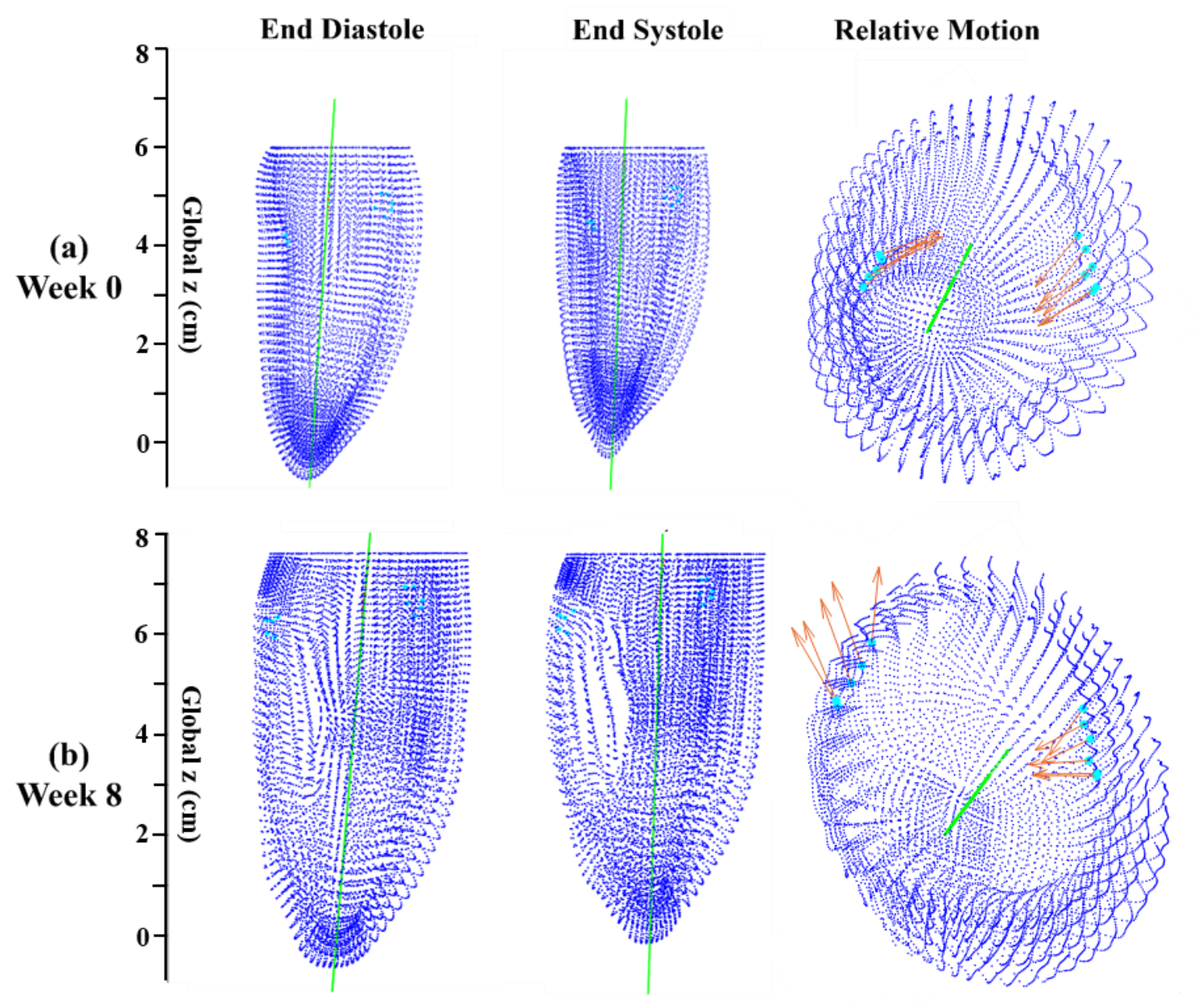

Figure 2.11 Three-dimensional node location (blue), CT node displacement vectors (orange) from end diastole to end systole, and LV long axis (green) calculated in MATLAB for the same animal at 0 week (a) and 8 week (b) timepoints. Global $\mathrm{z}$ axis is shown for both end diastolic and end systolic figures but does not apply to the relative motion figure. 
For each CT attachment node, the positive longitudinal direction was defined as the positive vertical axis relative to the FE model and the positive radial direction was defined as the outward vector pointing away from the long axis of the ventricle toward the corresponding CT attachment node. The circumferential direction was defined as the cross product between the longitudinal and radial vectors. CT attachment node displacements from each region (remote or infarct) were averaged to obtain radial, circumferential, and longitudinal displacement values for each papillary muscle within each animal at each timepoint. Circumferential, longitudinal, and radial local coordinate system vectors for one of the CT attachment nodes can be seen in Figure 2.12. 


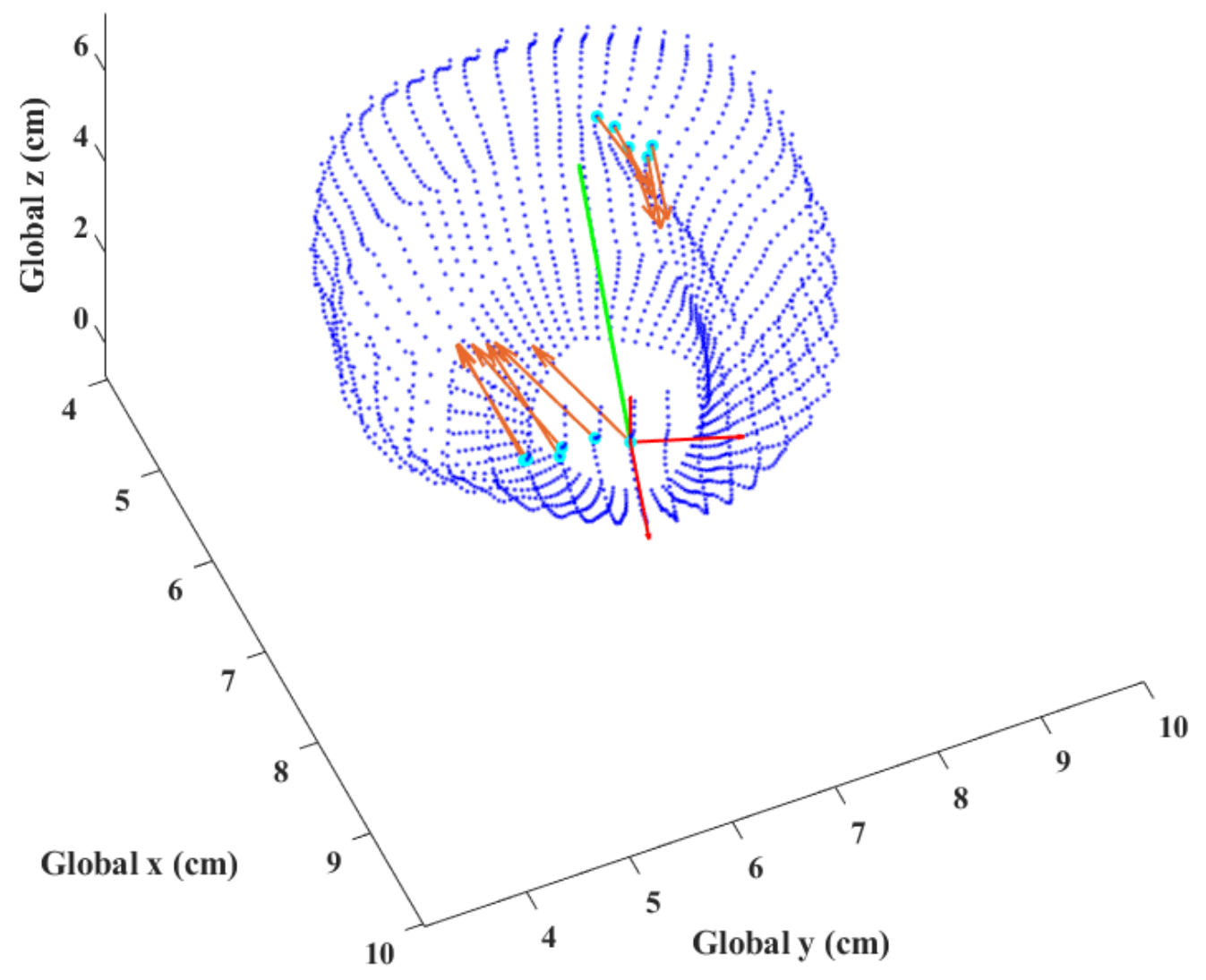

Figure 2.12 End diastolic LV endocardial nodes (dark blue) for one model highlighting CT attachment node locations (light blue), calculated long axis of the ventricle (green), CT attachment node motion vectors (orange) and local coordinate system for one of the CT attachment nodes (red). 


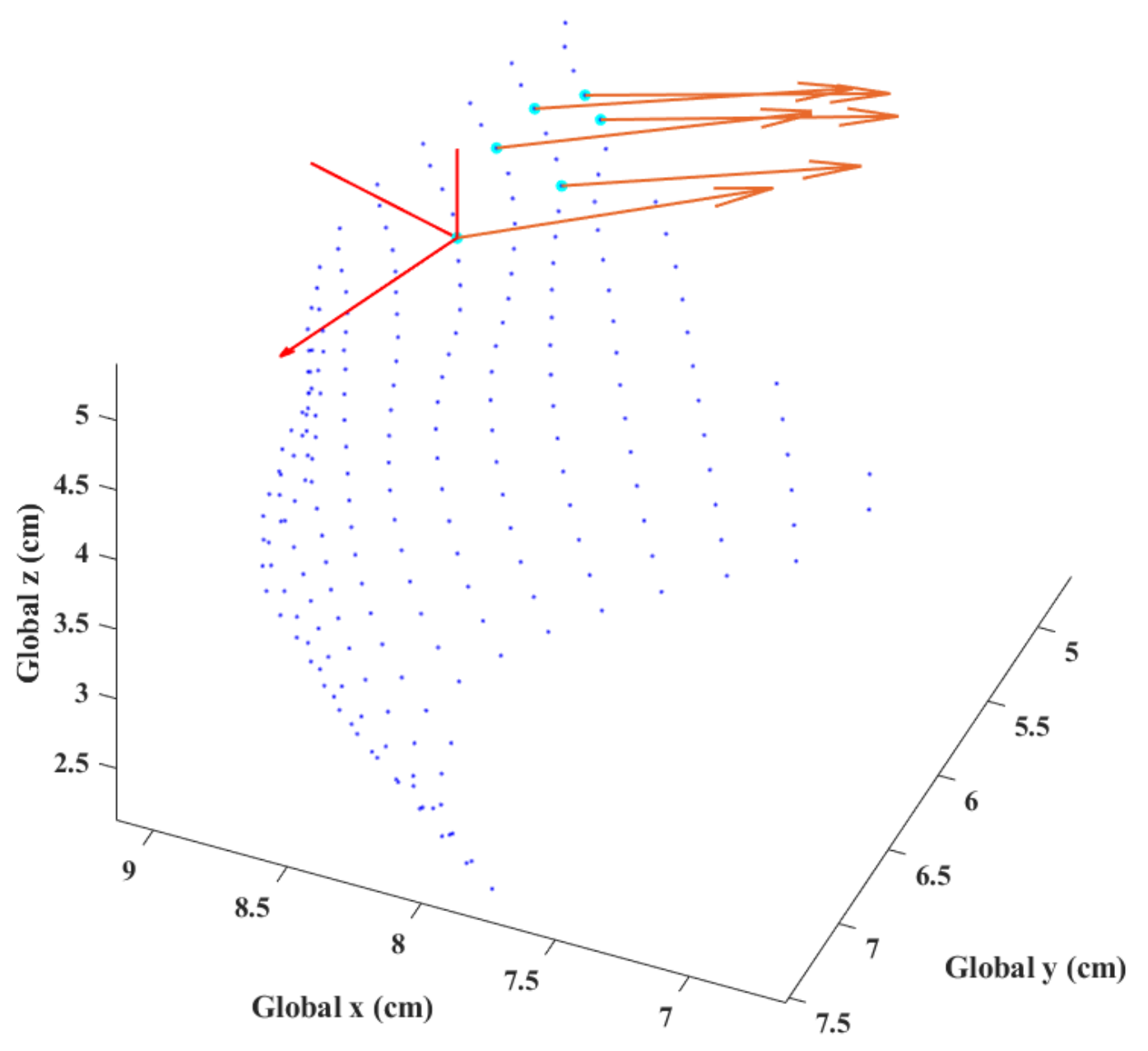

Figure 2.13 Closeup view of a small portion of the LV endocardial node cloud (dark blue) highlighting papillary CT attachment nodes (light blue), motion vectors (orange), and local coordinate system for one CT attachment node (red).

A repeated-measures ANOVA model was used to calculate test statistics and p-values for each term in the 3-way full-factorial model, considering the 3-way interaction between timepoint, region, and treatment, as well as each 2-way interaction and main effect. Pairwise differences were extracted and reported for effects that were statistically 
significant. Average displacements for each group, and timepoint for both tissue regions are provided in Section 3.1 of this document.

\subsection{Mitral Regurgitation}

As part of the original data collection and analysis by Rodell et al., mitral regurgitation (MR) was rated on a scale from 0-3 (none to severe) by two-dimensional echocardiography at both 0 and 8 week timepoints. Assessments of mitral regurgitation by two-dimensional echocardiography are shown in Figure 2.14 and Figure 2.15.

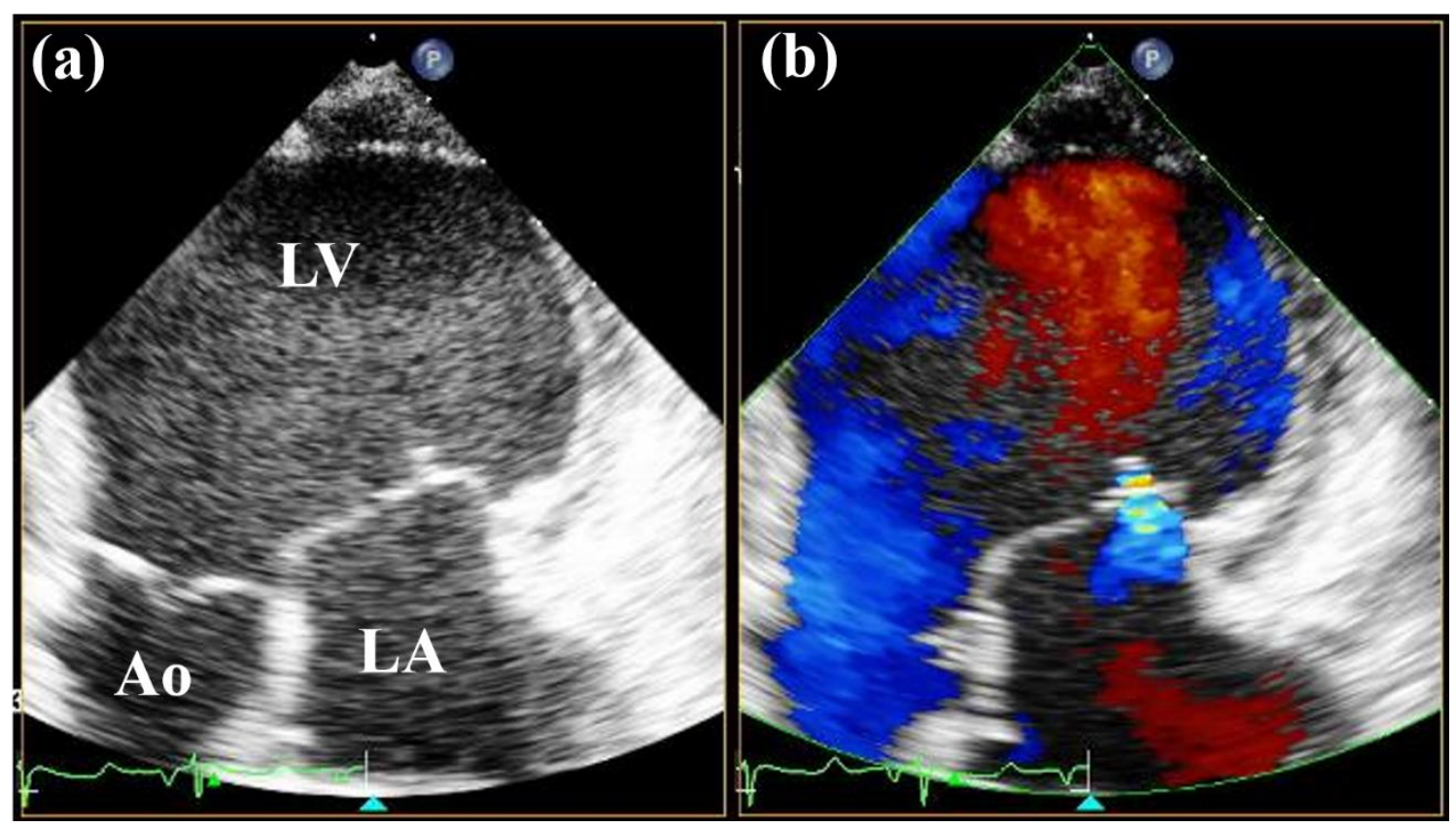

Figure 2.14 Two-dimensional echo apical view of a sheep heart after myocardial infarction showing (a) the left ventricle (LV), left atrium (LA), and aorta LV outflow tract (Ao); (b) color Doppler overlay of the same view showing an MR rating of $1+$. 


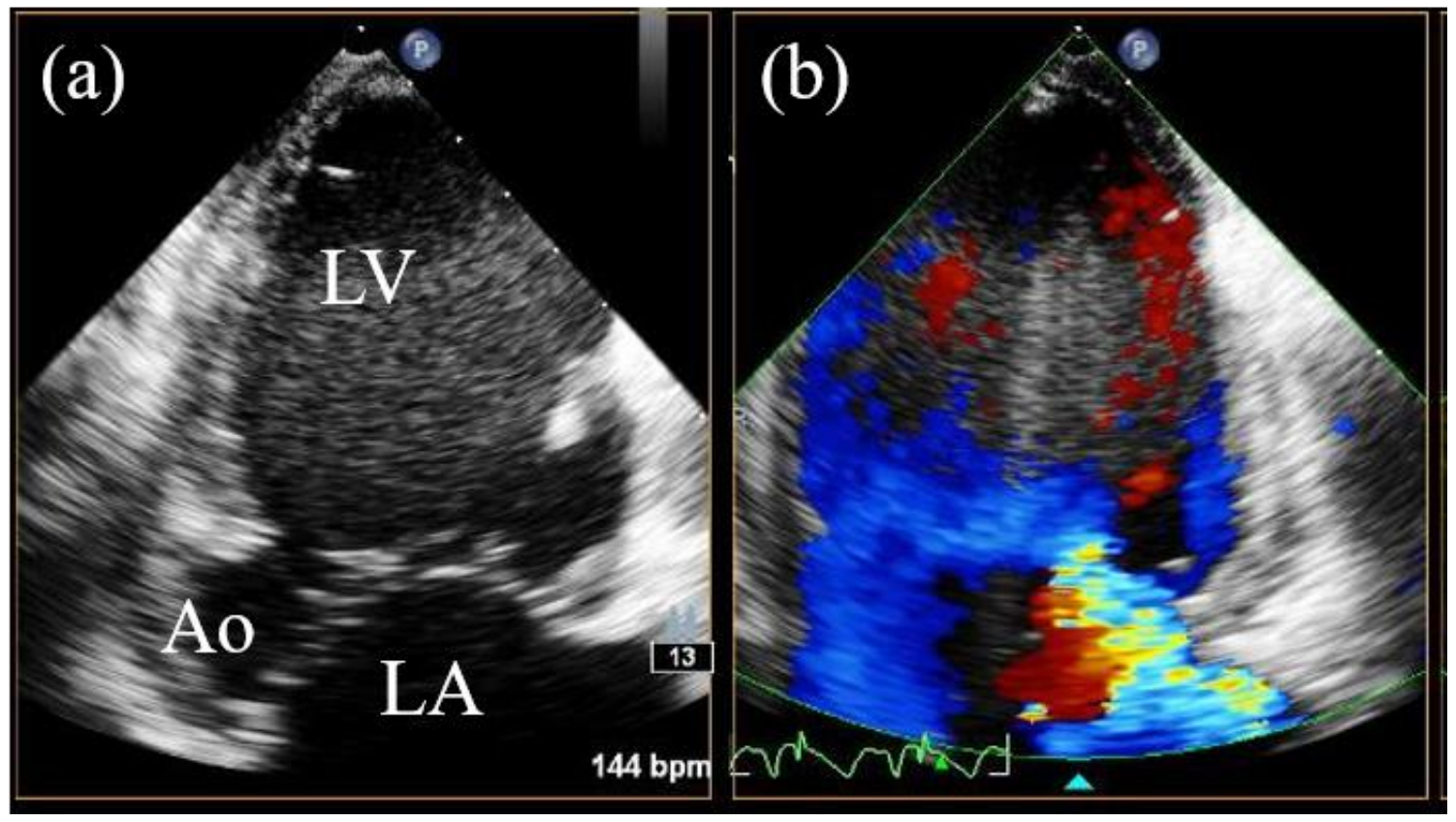

Figure 2.15 Two-dimensional echo apical view of a sheep heart after myocardial infarction showing (a) the left ventricle (LV), left atrium (LA), and aorta LV outflow tract (Ao); (b) color Dopper overlay of the same view showing an MR rating of 3+.

Additional quantification of MR was performed using short-axis MRI contours of the LV and RV. Full LV and RV end diastolic and end systolic contours were generated for each animal at both 0 week and 8 week timepoints in a semi-automated fashion using a custom MATLAB script. Ventricle volumes were calculated as the sum of voxel volumes contained within the set of contours for each ventricle. The stroke volume of each ventricle was calculated as the difference in ventricle volume between end diastole and end systole. Regurgitant volume and fraction were calculated from the ventricular stroke volumes based on methods described in previous studies [16, 21]. Regurgitant volume, $V_{R}$, was calculated as the difference between the LV and RV stroke volume: 
$V_{R}=S V_{L V}-S V_{R V}$

and the regurgitant fraction, $F_{R}$, was calculated as:

$F_{R}=V_{R} / S V_{L V}$

where $V_{R}$ is the regurgitant volume and $\mathrm{SV}$ units are in $\mathrm{mL}$. A comparison between MRI estimates of regurgitant volume, regurgitant fraction, and MR rating by two-dimensional echocardiography is shown in the results section of this thesis in Table 3.3. Average values for regurgitant volume and regurgitant fraction within each treatment group and timepoint are shown in the results section of this thesis in Table 3.4.

In order to quantify changes in ventricle size between timepoints, the average midventricle diameter was calculated for each model. The mid-ventricle slice was calculated as the slice halfway between the first and last slice on which the full LV contours were drawn. A circle was fit by minimization of the residual sum of squares to the LV contour on the mid ventricle slice, as well as the slice immediately below and above, and circle diameter was averaged between the three slices to approximate mid-LV diameter. Average mid-LV diameter at end diastole and end systole for each group and timepoint are provided in the results section of this thesis in Table 3.5. 


\section{CHAPTER 3. RESULTS}

\subsection{Papillary Muscle Motion}

Papillary muscle displacements in each region (remote and infarct), timepoint (0 and 8 weeks), and group (untreated MI and treated DC) are reported in this section. As previously described in Section 2.3, CT attachment node displacements from each region (remote or infarct) were averaged to obtain radial, circumferential, and longitudinal displacement values for each papillary muscle. Average papillary muscle displacements for the remote tissue region are reported in Table 3.1. It can be seen that the magnitude of inward radial displacement is significantly greater in the DC treatment group compared to the MI control group, at both the 0 week and 8 week timepoint. Furthermore, circumferential displacement in the remote tissue region is preserved relative to the 0 week timepoint in the DC treatment group, but is significantly less in the MI control group at the 8 week timepoint.

Table 3.1 Average left ventricle papillary muscle displacement in the remote tissue region. Data are presented as $\mathrm{mm} \pm$ standard deviation.

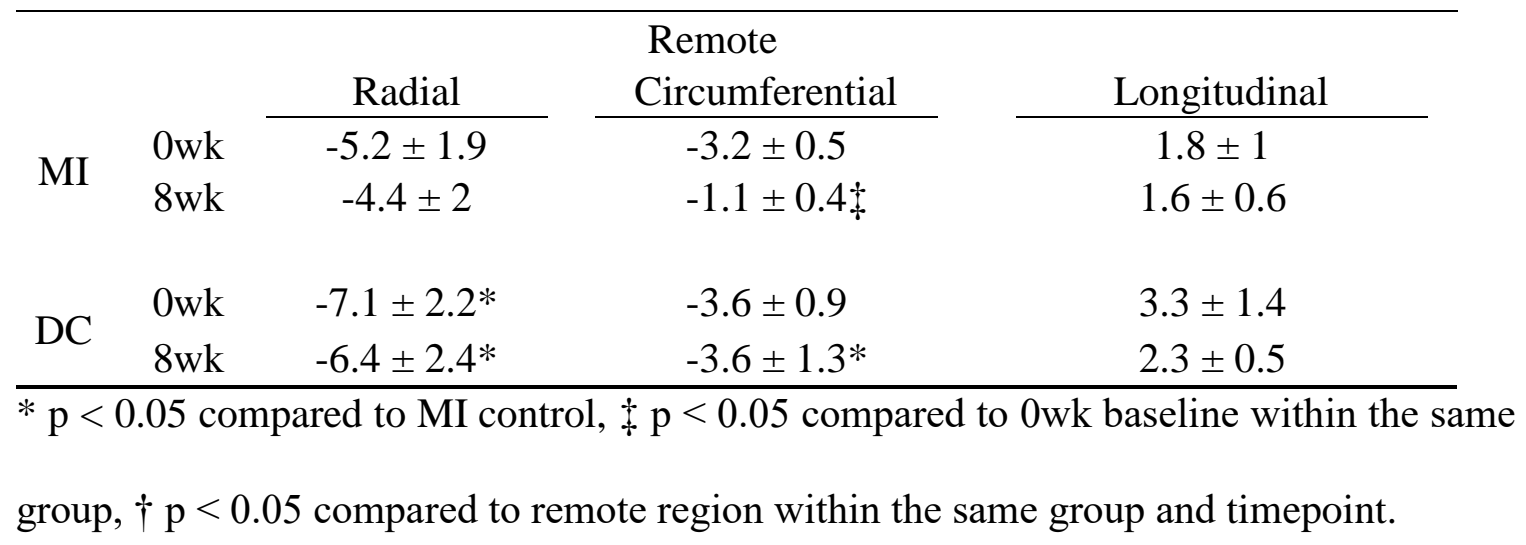


Average papillary muscle displacements for the infarct tissue region are provided in Table 3.2. Radial displacement in the infarct tissue region is significantly greater in the DC treatment group compared to the MI control group, at both timepoints. Additionally, average radial displacement in the infarct tissue region is positive for the untreated MI group, indicating outward movement of the infarcted myocardium during systole, while the direction of motion inward was preserved in the treated DC group. Circumferential displacement in the infarct tissue region decreased significantly from 0 weeks to 8 weeks in both animal groups. Longitudinal displacement in the infarct tissue region was significantly less at the 8 week timepoint in both groups compared to the 0 week timepoint.

Table 3.2 Average left ventricle papillary muscle displacement in the infarct tissue region. Data are presented as $\mathrm{mm} \pm$ standard deviation.

\begin{tabular}{|c|c|c|c|c|}
\hline \multicolumn{5}{|c|}{ Infarct } \\
\hline \multirow{3}{*}{ MI } & & Radial & Circumferential & Longitudinal \\
\hline & 0wk & $-5.3 \pm 1.1$ & $-3 \pm 0.9$ & $3.3 \pm 0.3$ \\
\hline & $8 w k$ & $1 \pm 0.8+\dagger$ & $-1.5 \pm 0.6 t$ & $1.9 \pm 0.8 \ddagger$ \\
\hline \multirow{2}{*}{$\mathrm{DC}$} & Owk & $-6.9 \pm 1.8^{*}$ & $-3.1 \pm 1.2$ & $4.4 \pm 0.7$ \\
\hline & $8 w k$ & $-1.3 \pm 1.4^{*}+\dagger$ & $-1.7 \pm 0.3+\dagger$ & $1.4 \pm 0.7 \ddagger$ \\
\hline
\end{tabular}

\subsection{Mitral Regurgitation}

MRI estimates of mitral regurgitation were compared to echo Doppler ratings by the methods previously discussed in Section 2.4. Table 3.3 shows the comparison of echo rating, MRI regurgitant volume, and MRI regurgitant fraction. Mitral regurgitation was 
rated at the 0 week and 8 week timepoint in each animal. Table 3.3 includes the number of times (n) that a specific echo Doppler rating was assigned across all animals and timepoints.

Table 3.3 Comparison between MRI estimates of regurgitant volume, regurgitant fraction, and rating by two-dimensional echocardiography. Data are presented as mean \pm standard deviation.

\begin{tabular}{ccccc}
\hline $\begin{array}{c}\text { Echo } \\
\text { Rating }\end{array}$ & & $\mathrm{n}$ & $\begin{array}{c}\text { MRI Regurgitant Volume } \\
(\mathrm{mL})\end{array}$ & $\begin{array}{c}\text { MRI Regurgitant } \\
\text { Fraction }(\%)\end{array}$ \\
\cline { 1 - 1 } & 5 & $2.4 \pm 2.5$ & $4.6 \pm 3.9$ \\
1 & 12 & $4.5 \pm 2.6$ & $8.7 \pm 3.9$ \\
3 & 5 & $9 \pm 4.2$ & $20.3 \pm 8.6$ \\
\hline
\end{tabular}

A comparison of MRI estimates of regurgitant volume and regurgitant fraction between treatment groups for each timepoint is shown in Table 3.4. Regurgitant volume and regurgitant fraction significantly increased in the untreated MI control group from the 0 week timepoint to the 8 week timepoint. Regurgitant volume and regurgitant fraction did not change from the 0 week to the 8 week timepoint in the treated DC group. In addition, these values are associated with echo ratings which imply no leakage of the valve at the 8 week timepoint. 
Table 3.4 Comparison of regurgitant volume and regurgitant fraction between treatment groups. Data are presented as mean \pm standard deviation.

\begin{tabular}{llccc}
\hline & & $0 \mathrm{wk}$ & & $8 \mathrm{wk}$ \\
\cline { 3 - 3 } Regurgitant Volume (mL) & MI & $2.7 \pm 1.3$ & & $14.8 \pm 12.2 *$ \\
& DC & $4.4 \pm 3.9$ & & $5.0 \pm 3.1^{*}$ \\
\multirow{2}{*}{ Regurgitant Fraction (\%) } & MI & $7.5 \pm 3.8$ & & $25.7 \pm 13.6 \%$ \\
& DC & $8.9 \pm 4.2$ & & $7.8 \pm 4.2^{*}$ \\
\hline
\end{tabular}

$* \mathrm{p}<0.05$ compared to MI control, $\$ \mathrm{p}<0.05$ compared to $0 \mathrm{wk}$ baseline within the same group.

A comparison of average mid-ventricle end diastolic and end systolic diameter between treatment groups for each timepoint is shown in Table 3.5. End diastolic diameter and end systolic diameter significantly increased from the 0 week timepoint to the 8 week timepoint in both the untreated MI and the treated DC animal groups. While there is no significant difference in end diastolic diameter between treatment groups, end systolic diameter is significantly greater in the untreated MI group than the treated DC group at the 8 week timepoint. This indicates that there is less dilation and greater inward radial motion at end systole in the treated animal group. 
Table 3.5 Comparison of average mid-LV diameter at end diastole and end systole between groups and timepoints. Data are presented as mean \pm standard deviation.

\begin{tabular}{lccccc}
\hline & & & $0 \mathrm{wk}$ & & $8 \mathrm{wk}$ \\
\cline { 5 - 5 } End Diastolic Diameter (mm) & MI & & $35 \pm 2$ & & $50.7 \pm 6.5 \dagger$ \\
& DC & & $36.2 \pm 5.2$ & & $44.6 \pm 4.3 \dagger$ \\
& & & & \\
End Systolic Diameter (mm) & MI & $21.8 \pm 3.2$ & & $42.4 \pm 6.8 \dagger$ \\
& DC & $21.1 \pm 5.4$ & & $33.4 \pm 5.5^{*} \dagger$ \\
\hline
\end{tabular}

$* \mathrm{p}<0.05$ compared to MI control, $\uparrow \mathrm{p}<0.05$ compared to $0 \mathrm{wk}$ baseline within the same group 


\section{CHAPTER 4. DISCUSSION}

While the relationship between the progression of ischemic mitral regurgitation and left ventricular remodeling after myocardial infarction has been investigated in depth, changes in quantifiable metrics of the ventricular wall and papillary muscle motion are not as readily available. Previous research has shown that chordae tendineae (CT) have an important role in mitral valve competence as well as left ventricular geometry and function $[10,18]$. Due to tethering between the mitral valve and the papillary muscles in the left ventricle via $\mathrm{CT}$, it is plausible that changes in left ventricular wall and papillary muscle motion following myocardial infarction could contribute to the progression of ischemic mitral regurgitation [10, 11, 17, 21 35]. Previous research from Gorman et al. concluded that myocardial infarctions that include the anterior papillary muscle do not produce mitral regurgitation regardless of size, while mitral regurgitation was shown to occur following an infarct involving the posterior papillary muscle. According to Gorman et al., large posterior infarcts may result in mitral regurgitation caused in part by discoordination of papillary muscle relationships to the mitral valve [11]. The current study presents a method to quantify changes in papillary muscle motion following posterolateral myocardial infarction which may contribute to the development and progression of ischemic mitral regurgitation.

In this study, quantifiable changes in CT papillary muscle attachment site displacements were assumed to be representative of overall papillary muscle motion. Changes in papillary muscle motion and estimates of mitral regurgitation were compared between a group of animals that received a hydrogel treatment (DC) shown to limit ventricular remodeling after myocardial infarction and a control group (MI). Regurgitant 
volume and regurgitant fraction calculated from MRI estimates of LV and RV stroke volume (Table 3.4) were not different between the DC and MI groups at the 0 week baseline timepoint. Both regurgitant volume and regurgitant fraction significantly increased in the untreated MI group from the 0 week to 8 week timepoint, while no significant change was observed in the treated DC group. Average CT papillary muscle attachment node displacement in the radial, circumferential, and longitudinal directions were compared between treatment groups (MI, DC), region (Remote, Infarct), and timepoint (0 week, 8 week) as shown in Table 3.1 and Table 3.2.

The magnitude of radial displacement was significantly greater in the treated DC group than the untreated MI group regardless of timepoint or region. Despite this systematic difference between groups, it is important to note that the calculated outward radial displacement of the papillary muscle in the infarct region is indicative of an outward bulging of the compromised infarcted tissue region at the 8-week timepoint in the untreated MI group. This outward radial displacement calculated from the FE model simulation is consistent with recorded observations of left ventricular dyskinesia from MRI and echocardiography. The mechanism for development of mitral regurgitation from dyskinesis of the posterior portion of the LV wall is postulated in Godley et al. and Gorman et al $[10,11]$. A posterior displacement of the LV wall that occurs in dyskinesis could increase the tension on the chordae tendineae connecting the posterior papillary muscle and the anterior leaflet of the mitral valve, causing the anterior leaflet to be pulled into the LV cavity. A similar mechanism for the development of MR is outline in a more recent study by Levine et al [21]. 
The Rodell paper that originally reported on the efficacy of this specific hydrogel treatment to prevent left ventricular remodeling did not comment on the development of mitral regurgitation. A previous study from McGarvey et al. under the guidance of the Gorman group showed that injectable microsphere gels improved ratings of mitral regurgitation by echocardiography in a porcine model [24]. The work presented in this thesis provides additional insight into the development of mitral regurgitation following posterolateral myocardial infarction by providing quantifiable metrics of changes in papillary muscle movement. While it is possible that future studies may be able to obtain papillary muscle motion experimentally using 3D displacement-encoded MRI, the appropriate MRI data to perform this type of analysis was not collected in the present study. This thesis outlines an effective method for quantification of papillary muscle motion using 3D FE models built from a stack of 2D cine MR images.

Mid-LV diameter was calculated to provide a metric that could be used to gain additional insight on both global LV remodeling as well as changes in papillary muscle motion. As can be seen in Table 3.5, end diastolic diameter and end systolic diameter were not different between the MI control group and the treated DC group at the 0 week timepoint. At the 8 week timepoint, the end diastolic diameter was not significantly different between the two groups; however, average mid-LV end systolic diameter was significantly less in the treated DC group than in the untreated MI group. Due to an increase in regurgitant volume in the MI group that was not observed in the treated DC group, similarity in end diastolic diameter between groups at the 8 week timepoint could suggest that inability to maintain a low systolic diameter relative to end diastolic diameter is a predictor of mitral regurgitation. Jensen et al. found that anterior to posterior papillary 
muscle distance at end systole showed the strongest correlation with regurgitant volume of papillary muscle position parameters that were tested, but did not discuss combinations of effects [17]. The results of the current study indicate that the DC treatment has both a global effect, indicated by the smaller end systolic diameter, as well as a local effect, indicated by the inward radial motion of the infarcted papillary muscle region.

The magnitude of circumferential displacement significantly decreased between 0 week and 8 week timepoints in the infarct region in both groups, as well as the remote region in the untreated MI group. Circumferential displacement was preserved between 0 and 8 weeks in the remote region of the treated DC group. Longitudinal displacements similarly decreased in the infarct region between 0 and 8 weeks for both treated and untreated groups. Although the magnitude of displacement decreases in all directions in the infarct region for both the MI and DC groups, the positive (outward) motion in the radial direction of the MI group could contribute to the significant increase in regurgitant volume. Additionally, circumferential displacement, which is linked to the twisting motion of the LV, is decreased in both the remote and infarct region of the MI group but is preserved in the remote region of the DC group. The combination of these two factors may serve to highlight the role of changes in papillary muscle motion in the progression of mitral regurgitation. A correlation analysis between measurements of mitral regurgitation and changes in direction and magnitude of displacement may provide some additional insight into the results outlined in this study. Regardless of the underlying mechanism, the results of this study show that regurgitant volume did not increase 8 weeks after myocardial infarction in the group of animals that received the hydrogel injection therapy in contrast to the significant increase in regurgitant volume observed in the untreated animal group. 


\section{CHAPTER 5. CONCLUSION}

The purpose of this study, within the scope of this thesis, was to quantify the differences in papillary muscle motion and progression of mitral regurgitation between a group of untreated animals and a group of animals treated with hydrogel injection therapy. As outlined in this thesis, dyskinesis of the LV wall involving one or both papillary muscles is thought to influence the development of mitral regurgitation due to tethering between the mitral valve and papillary muscles via the chordae tendineae. Early research used echocardiography to estimate LV myocardium dyskinesis involving the papillary muscles. A more recent study employed the use of 3D morphology cardiac MRI to characterize the spatial location of the papillary muscles in groups of animals with and without FIMR [17]. The current study used FE model simulations constructed from LV contours to show that while differences in papillary muscle motion occur between 0 and 8 weeks post-MI in both treated and untreated groups, dyskinesis of the posterior papillary muscle thought to contribute to MR was only observed in the untreated group of animals.

Estimates of mitral regurgitation from MRI showed a significant increase in regurgitant volume in the untreated group of animals post-MI while regurgitant volume did not increase in treated animals. Mid-ventricle diameter increased significantly in both the treated and untreated groups from 0 to 8 weeks post-MI and there was no difference in end diastolic diameter between the two groups at 8 weeks; however, mid-LV end systolic diameter was significantly lower in the treated group than in the untreated group. Lower systolic diameter in the treated group at 8 weeks post-MI may suggest that systolic motion from 0 weeks to 8 weeks was preserved better in animals that received the hydrogel treatment than in those that did not. 
Existing treatment for limiting mitral regurgitation and posterior LV dyskinesia as a result of myocardial infarction includes methods such as reinforcement of the wall via patch device, balloon inflation, or suture rows [21]. Hydrogel injection therapy has been shown to limit LV remodeling and development of MR 8 weeks post-MI and could serve as a minimally invasive treatment alternative. This thesis presented methods to quantify changes in papillary muscle motion after myocardial infarction using FE models built from 2D short-axis LV MR images. In future work, a comparison study between motion data obtained using animal specific FE models as presented in this thesis and motion data obtained from 3D DENSE MRI could provide important insights into these results. In order to better understand the development of mitral regurgitation using the methods presented here, future research could focus on the development and implementation of a model that encompasses the entire LV including mitral valve and chordae tendineae in order to simulate the tethering interaction throughout the cardiac cycle. 


\section{REFERENCES}

[1] Ahmed, Enas M. "Hydrogel: Preparation, Characterization, and Applications: A Review." Journal of Advanced Research, vol. 6, no. 2, 18 July 2013, pp. 105121., doi:10.1016/j.jare.2013.07.006.

[2] Birnbaum, Yochai, et al. "Mitral Regurgitation Following Acute Myocardial Infarction.” Coronary Artery Disease, vol. 13, no. 6, 2002, pp. 337-344., doi:10.1097/00019501-200209000-00006.

[3] Bursi, Francesca, et al. "Mitral Regurgitation After Myocardial Infarction: A Review." The American Journal of Medicine, vol. 119, no. 2, Feb. 2006, pp. $103-$ 112., doi:10.1016/j.amjmed.2005.08.025.

[4] “Chapter 12: The Cardiovascular System: The Heart.” Essentials of Anatomy \&amp; Physiology, by Frederic Martini and Edwin F. Bartholomew, Pearson Education, Inc., 2017.

[5] Costa, K. D., et al. "Modelling Cardiac Mechanical Properties in Three Dimensions." Philosophical Transactions of the Royal Society of London. Series A: Mathematical, Physical and Engineering Sciences, vol. 359, no. 1783, 2001, pp. 1233-1250., doi:10.1098/rsta.2001.0828.

[6] Dorsey, Shauna M., et al. "MRI Evaluation of Injectable Hyaluronic Acid-Based Hydrogel Therapy to Limit Ventricular Remodeling after Myocardial Infarction." Biomaterials, vol. 69, 2015, pp. 65-75., doi:10.1016/j.biomaterials.2015.08.011.

[7] Dujardin, Karl S., et al. "Grading of Mitral Regurgitation by Quantitative Doppler Echocardiography." Circulation, vol. 96, no. 10, 18 Nov. 1997, pp. 3409-3415., doi:10.1161/01.cir.96.10.3409.

[8] Ge, Liang, et al. "Moderate Ischemic Mitral Regurgitation After Posterolateral Myocardial Infarction in Sheep Alters Left Ventricular Shear but Not Normal Strain in the Infarct and Infarct Borderzone." The Annals of Thoracic Surgery, vol. 101, no. 5, 2016, pp. 1691-1699., doi:10.1016/j.athoracsur.2015.10.083.

[9] Gibas, Iwona, and Helena Janik. "Review: Synthetic Polymer Hydrogels For Biomedical Applications." Chemistry and Chemical Technology, vol. 4, no. 4, Oct. 2010.

[10] Godley, R W, et al. "Incomplete Mitral Leaflet Closure in Patients with Papillary Muscle Dysfunction.” Circulation, vol. 63, no. 3, Mar. 1981, pp. 565-571., doi:10.1161/01.cir.63.3.565.

[11] Gorman, Joseph H., et al. "Infarct Size And Location Determine Development Of Mitral Regurgitation In The Sheep Model.” The Journal of Thoracic and Cardiovascular Surgery, vol. 115, no. 3, 1998, pp. 615-622., doi:10.1016/s00225223(98)70326-5. 
[12] Grigioni, F, et al. "Ischemic Mitral Regurgitation. Long-Term Outcome and Prognostic Implications with Quantitative Doppler Assessment.” ACC Current Journal Review, vol. 10, no. 5, 2001, p. 33., doi:10.1016/s1062-1458(01)00387-7.

[13] Guccione, J. M., and A. D. McCulloch. "Mechanics of Active Contraction in Cardiac Muscle: Part I-Constitutive Relations for Fiber Stress That Describe Deactivation.” Journal of Biomechanical Engineering, vol. 115, no. 1, 1993, p. 72., doi:10.1115/1.2895473.

[14] Guccione, J. M., et al. "Mechanics of Active Contraction in Cardiac Muscle: Part II - Cylindrical Models of the Systolic Left Ventricle." Journal of Biomechanical Engineering, vol. 115, no. 1, Feb. 1993, p. 82., doi:10.1115/1.2895474.

[15] Guccione, J. M., et al. "Passive Material Properties of Intact Ventricular Myocardium Determined From a Cylindrical Model." Journal of Biomechanical Engineering, vol. 113, no. 1, Feb. 1991, p. 42., doi:10.1115/1.2894084.

[16] Hundley, W. Gregory, et al. "Magnetic Resonance Imaging Assessment of the Severity of Mitral Regurgitation.” Circulation, vol. 92, no. 5, Sept. 1995, pp. 1151-1158., doi:10.1161/01.cir.92.5.1151.

[17] Jensen, Henrik, et al. "Three-Dimensional Assessment of Papillary Muscle Displacement in a Porcine Model of Ischemic Mitral Regurgitation.” The Journal of Thoracic and Cardiovascular Surgery, vol. 140, no. 6, Mar. 2010, pp. 13121318., doi:10.1016/j.jtcvs.2009.12.042.

[18] Kon, MW. The Journal of Heart Valve Disease, vol. 13, no. 4, July 2004, pp. 600-607.

[19] Kong, Fanwei, et al. "Finite Element Analysis of Annuloplasty and Papillary Muscle Relocation on a Patient-Specific Mitral Regurgitation Model.” Plos One, vol. 13, no. 6, 2018, doi:10.1371/journal.pone.0198331.

[20] Lamas, GA. “Clinical Significance of Mitral Regurgitation after Acute Myocardial Infarction. Survival and Ventricular Enlargement Investigators." Circulation, vol. 96, no. 3, 5 Aug. 1997, pp. 827-833.

[21] Levine, Robert A., and Ehud Schwammenthal. "Ischemic Mitral Regurgitation on the Threshold of a Solution." Circulation, vol. 112, no. 5, 2005, pp. 745-758., doi:10.1161/circulationaha.104.486720.

[22] Marzilli, M., et al. "Role of the Papillary Muscle in Opening and Closure of the Mitral Valve." American Journal of Physiology-Heart and Circulatory Physiology, vol. 238, no. 3, Mar. 1980, doi:10.1152/ajpheart.1980.238.3.h348.

[23] Mcgarvey, Jeremy R., et al. "Injectable Microsphere Gel Progressively Improves Global Ventricular Function, Regional Contractile Strain, and Mitral Regurgitation After Myocardial Infarction.” The Annals of Thoracic Surgery, vol. 99, no. 2, 2015, pp. 597-603., doi:10.1016/j.athoracsur.2014.09.014.

[24] Mcgarvey, Jeremy R., et al. “Temporal Changes in Infarct Material Properties: An In Vivo Assessment Using Magnetic Resonance Imaging and Finite Element 
Simulations." The Annals of Thoracic Surgery, vol. 100, no. 2, 2015, pp. $582-$ 589., doi:10.1016/j.athoracsur.2015.03.015.

[25] Obadia, Jean F., et al. "Mitral Subvalvular Apparatus." Circulation, vol. 96, no. 9, 1997, pp. 3124-3128., doi:10.1161/01.cir.96.9.3124.

[26] Orlic, Donald, et al. "Bone Marrow Stem Cells Regenerate Infarcted Myocardium.” Pediatric Transplantation, vol. 7, 2003, pp. 86-88., doi:10.1034/j.1399-3046.7.s3.13.x.

[27] Rodell, Christopher B., et al. "Injectable Shear-Thinning Hydrogels for Minimally Invasive Delivery to Infarcted Myocardium to Limit Left Ventricular

Remodeling." Circulation: Cardiovascular Interventions, vol. 9, no. 10, Oct. 2016, doi:10.1161/circinterventions.116.004058.

[28] Rodriguez, F. "Importance of Mitral Valve Second-Order Chordae for Left Ventricular Geometry, Wall Thickening Mechanics, and Global Systolic Function." Circulation, vol. 110, no. 11_suppl_1, 2004, doi:10.1161/01.cir.0000138580.57971.b4.

[29] Soleimani, Mehrdad, et al. "Moderate Mitral Regurgitation Accelerates Left Ventricular Remodeling After Posterolateral Myocardial Infarction." The Annals of Thoracic Surgery, vol. 92, no. 5, Nov. 2011, pp. 1614-1620., doi:10.1016/j.athoracsur.2011.05.117.

[30] Sutton, Martin G. St. John, and Norman Sharpe. "Left Ventricular Remodeling After Myocardial Infarction." Circulation, vol. 101, no. 25, 2000, pp. 2981-2988., doi:10.1161/01.cir.101.25.2981.

[31] Thygesen, Kristian, et al. "Universal Definition of Myocardial Infarction." Journal of the American College of Cardiology, vol. 50, no. 22, 2007, pp. 21732195., doi:10.1016/j.jacc.2007.09.011.

[32] Veronesi, Federico, et al. "Quantification of Mitral Apparatus Dynamics in Functional and Ischemic Mitral Regurgitation Using Real-Time 3-Dimensional Echocardiography." Journal of the American Society of Echocardiography, vol. 21, no. 4, 2008, pp. 347-354., doi:10.1016/j.echo.2007.06.017.

[33] Wang, Hua, et al. "Effects of Hydrogel Injection on Borderzone Contractility Post-Myocardial Infarction." Biomechanics and Modeling in Mechanobiology, vol. 17, no. 5, 2018, pp. 1533-1542., doi:10.1007/s10237-018-1039-2.

[34] Wang, Hua, et al. "Computational Investigation of Transmural Differences in Left Ventricular Contractility." Journal of Biomechanical Engineering, vol. 138, no. 11, 2016, p. 114501., doi:10.1115/1.4034558.

[35] Wenk, Jonathan F., et al. "First Finite Element Model of the Left Ventricle With Mitral Valve: Insights Into Ischemic Mitral Regurgitation.” The Annals of Thoracic Surgery, vol. 89, no. 5, May 2010, pp. 1546-1553., doi:10.1016/j.athoracsur.2010.02.036. 
[36] Wenk, Jonathan F., et al. "A Method for Automatically Optimizing Medical Devices for Treating Heart Failure: Designing Polymeric Injection Patterns." Journal of Biomechanical Engineering, vol. 131, no. 12, 2009, p. 121011., doi:10.1115/1.4000165.

[37] Wenk, Jonathan F., et al. "Regional Left Ventricular Myocardial Contractility and Stress in a Finite Element Model of Posterobasal Myocardial Infarction." Journal of Biomechanical Engineering, vol. 133, no. 4, 2011, p. 044501., doi:10.1115/1.4003438.

[38] Yin F C, and L L Demer. "Passive Biaxial Mechanical Properties of Isolated Canine Myocardium.” The Journal of Physiology, vol. 339, no. 1, 1983, pp. 615630., doi:10.1113/jphysiol.1983.sp014738.

[39] Yiu, Siu F., et al. "Determinants of the Degree of Functional Mitral Regurgitation in Patients With Systolic Left Ventricular Dysfunction." Circulation, vol. 102, no. 12, 2000, pp. 1400-1406., doi:10.1161/01.cir.102.12.1400.

[40] Zeng, Xin, et al. "Efficacy of Polymer Injection for Ischemic Mitral Regurgitation." JACC: Cardiovascular Interventions, vol. 8, no. 2, 2015, pp. 355 363., doi:10.1016/j.jcin.2014.09.016.

[41] Zhang, Xiaoyan, et al. "Regional Quantification of Myocardial Mechanics in Rat Using 3D Cine DENSE Cardiovascular Magnetic Resonance." NMR in Biomedicine, vol. 30, no. 8, 2017, doi:10.1002/nbm.3733. 
VITA

\section{EDUCATION}

- University of Kentucky, Lexington, KY

- Bachelor of Science in Mechanical Engineering, May 2015

\section{PROFESSIONAL POSITIONS}

- NASA Johnson Space Center, Houston, TX

○ Engineer, September 2015 - Present

○ Intern, May 2014 - August 2014, May 2015 - August 2015

- Wenner-Gren Aeronautical Research Laboratory, Lexington, KY

○ Research Technician, May 2011 - May 2014

\section{HONORS AND AWARDS}

- National Aeronautics and Space Administration, Houston, TX

○ Johnson Space Center Group Achievement Award, December 2016

- Johnson Space Center Group Achievement Award, August 2015

- National Aeronautics and Space Administration, Mountain View, CA

- Ames Research Center Travel Grant Recipient, May 2012

- MEI Technologies, Houston, TX

- Sustained Total Achievement Award, December 2016

- University of Kentucky, Lexington, KY

○ Dean's List, Fall 2011 - Spring 2015

o Honors Program

- Kentucky Educational Excellence Scholarship

o Provost Scholarship

\section{PUBLICATIONS}

- Ferguson, CR, Gorman, RC, Wenk, JF. "Quantification of Papillary Muscle Motion and Mitral Regurgitation after Myocardial Infarction." Paper accepted for presentation at annual meeting of the Society for Experimental Mechanics, Reno, NV (2019).

- Ferguson, CR, Gorman, RC, Wenk, JF. “Quantification of Papillary Muscle Motion and Mitral Regurgitation after Myocardial Infarction.” Poster session presented at UK College of Engineering Biomedical Research Day, Lexington, KY (2018).

- Laurie, SS, Macias, BR, Ferguson, CR, Dunn, JT, Ebert, D, Lee, SMC, Dulchavsky, SA, Hargens, AR, Stenger, MB. "Submacular Choroid Thickness Increases During 
Long-Duration Spaceflight." Poster session presented at the annual meeting of the Association for Research in Vision and Ophthalmology, Honolulu, HI (2018).

- Ferguson, CR, Laurie, SS, Macias, BR, Ebert, DJ, Lee, SMC, Stenger, MB. "Quantification of choroidal folds in an astronaut completing long-duration spaceflight." Poster session presented at the annual meeting of the Association for Research in Vision and Ophthalmology, Baltimore, MD (2017).

- Laurie, S, Vizzeri, G, Taibbi, G, Ferguson, CR, Hu, X, Lee, S, Ploutz-Snyder, R, Smith, S, Zwart, S, Stenger, M. "Effects of Short-Term Mild Hypercapnia during HeadDown Tilt on Intracranial Pressure and Ocular Structures in Healthy Human Subjects." Physiological Reports (2017).

- Evans, JM, Ribeiro, LC, Moore, FB, Wang, S, Zhang, Q, Kostas, V, Ferguson, CR, Serrador, J, Falvo, M, Stenger, MB, Goswami, N, Rask, JC, Smith, JD, Knapp, CF. "Hypovolemic Men and Women Regulate Blood Pressure Differently Following Exposure to Artificial Gravity." European Journal of Applied Physiology, vol. 115, no. 12, 2015, pp. 2631-2640., doi:10.1007/s00421-015-3261-2.

- Ferguson, CR, Lee, SMC, Stenger, MB, Laurie, SS. "Measurement of acute changes in choroid thickness in healthy eyes during posture change using optical coherence tomography." NASA Technical Reports Server (2015).

- Ferguson, CR, Laurie, SS, Stenger, MB. "Modelflow Determination of Stroke Volume." Paper presented at NASA Johnson Space Center, Houston, TX (2014).

- Ferguson, CR, Lee, SMC, Stenger, MB, Platts, SH, Laurie, SS. "Modelflow Estimates of Stroke Volume Do Not Correlate With Doppler Ultrasound Estimates During Upright Posture." NASA Technical Reports Server (2014).

- Evans, JM, Ferguson, CR, Zhang, Q, Moore, FB, Smith, JD, Stenger, MB, Ribeiro, LC, Knapp, CF. "Mechanisms of Orthostatic Tolerance Improvement Following Artificial Gravity Exposure Differ between Men and Women." The Journal of the Federation of American Societies for Experimental Biology (2014).

- Evans, JM, Ribeiro, LC, Wang, S, Falvo, MJ, Serrador, J, Ferguson, CR, Moore, FB, Smith, JD, Rask, JC, Kostas, V, Zhang, Q, Knapp, CF. "Improvement of Hypovolemic Men's and Women's Orthostatic Tolerance by a Short Exposure to Artificial Gravity." The Journal of the Federation of American Societies for Experimental Biology (2013). 